TRANSACTIONS OF THE

AMERICAN MATHEMATICAL SOCIETY

Volume 358, Number 7, Pages 2965-3001

S 0002-9947(05)03787-6

Article electronically published on June 10, 2005

\title{
BESOV SPACES WITH NON-DOUBLING MEASURES
}

\author{
DONGGAO DENG, YONGSHENG HAN, AND DACHUN YANG
}

\begin{abstract}
Suppose that $\mu$ is a Radon measure on $\mathbb{R}^{d}$, which may be nondoubling. The only condition on $\mu$ is the growth condition, namely, there is a constant $C_{0}>0$ such that for all $x \in \operatorname{supp}(\mu)$ and $r>0$,

$$
\mu(B(x, r)) \leq C_{0} r^{n},
$$

where $0<n \leq d$. In this paper, the authors establish a theory of Besov spaces $\dot{B}_{p q}^{s}(\mu)$ for $1 \leq p, q \leq \infty$ and $|s|<\theta$, where $\theta>0$ is a real number which depends on the non-doubling measure $\mu, C_{0}, n$ and $d$. The method used to define these spaces is new even for the classical case. As applications, the lifting properties of these spaces by using the Riesz potential operators and the dual spaces are obtained.
\end{abstract}

\section{INTRODUCTION}

Suppose that $\mu$ is a Radon measure on $\mathbb{R}^{d}$, which may be non-doubling. The only condition on $\mu$ is the growth condition, namely, there is a constant $C_{0}>0$ such that for all $x \in \operatorname{supp}(\mu)$ and $r>0$,

$$
\mu(B(x, r)) \leq C_{0} r^{n},
$$

where $0<n \leq d$.

Our goal in this paper is to develop a theory of Besov spaces associated to nondoubling measures on $\mathbb{R}^{d}$.

It is well known that the doubling property of the underlying measure is a basic condition in the classical Calderón-Zygmund theory of harmonic analysis. Recently more attention has been paid to non-doubling measures. It has been shown that many results of this theory still hold without assuming the doubling property; see [16, 17, 18, 19, 23, 24, 25, 29, 5, 6] for some results on Calderón-Zygmund operators, [15, 26, 27, 28] for some other results related to the spaces $B M O(\mu)$ and $H^{1}(\mu)$, and $7,8,20$, for the vector-valued inequalities on the Calderón-Zygmund operators and weights.

Received by the editors June 17, 2003 and, in revised form, May 16, 2004.

2000 Mathematics Subject Classification. Primary 42B35; Secondary 46E35, 42B25, 47B06, 46B10, 43A99.

Key words and phrases. Non-doubling measure, Besov space, Calderón-type reproducing formula, approximation to the identity, Riesz potential, lifting property, dual space.

The first author's research was supported by NNSF (No. 10171111) of China.

The second author's research was supported by NNSF (No. 10271015) of China.

The third (corresponding) author's research was supported by NNSF (No. 10271015) and RFDP (No. 20020027004) of China.

(C)2005 American Mathematical Society Reverts to public domain 28 years from publication 
Let us first recall the definition of Besov spaces on $\mathbb{R}^{d}$. It is well known that the Littlewood-Paley theory gives a uniform treatment of function spaces on $\mathbb{R}^{d}$. Suppose that $\psi$ is a Schwartz function satisfying the following conditions:

(i) $\operatorname{supp} \widehat{\psi} \subset\left\{\xi \in \mathbb{R}^{d}: 1 / 2 \leq|\xi| \leq 2\right\}$;

(ii) $|\widehat{\psi}(\xi)| \geq C>0$ for all $3 / 5 \leq|\xi| \leq 5 / 3$.

The Besov space $\dot{B}_{p, q}^{s}\left(\mathbb{R}^{d}\right)$ is defined to be the set of all $f \in \mathcal{S}^{\prime}\left(\mathbb{R}^{d}\right) / \mathcal{P}\left(\mathbb{R}^{d}\right)$ such that for $-\infty<s<\infty$ and $0<p, q \leq \infty$,

$$
\|f\|_{\dot{B}_{p, q}^{s}\left(\mathbb{R}^{d}\right)}=\left\{\sum_{k=-\infty}^{\infty} 2^{s k q}\left\|\psi_{k} * f\right\|_{L^{p}\left(\mathbb{R}^{d}\right)}^{q}\right\}^{1 / q}<\infty
$$

where $\psi_{k}(x)=2^{k d} \psi\left(2^{k} x\right)$ for $x \in \mathbb{R}^{d}$ and $k \in \mathbb{Z}$, and $\mathcal{S}^{\prime}\left(\mathbb{R}^{d}\right) / \mathcal{P}\left(\mathbb{R}^{d}\right)$ is the space of Schwartz distributions modulo the space of all polynomials.

A key tool used to study these Besov spaces is the so-called Calderón reproducing formula which was first provided by Calderón in [1]. This formula says that for any given function $\psi$ satisfying the above conditions (i) and (ii), there exists a function $\phi$ with the properties similar to $\psi$ such that

$$
f=\sum_{k=-\infty}^{\infty} \phi_{k} * \psi_{k} * f
$$

where the series converges in $L^{2}\left(\mathbb{R}^{d}\right), \mathcal{S}\left(\mathbb{R}^{d}\right)$ and $\mathcal{S}^{\prime}\left(\mathbb{R}^{d}\right) / \mathcal{P}\left(\mathbb{R}^{d}\right)$; see 4 .

Using this formula one can show that the Besov spaces $\dot{B}_{p q}^{s}\left(\mathbb{R}^{d}\right)$ are independent of the choice of $\psi$. Also using this formula one can establish the embedding theorems, the interpolation theorems, duality, atomic decomposition and the $T 1$ theorems for the spaces $\dot{B}_{p q}^{s}\left(\mathbb{R}^{d}\right)$; see [4, 21, 31, 32, for more details.

By Coifman's ideas, David, Journé and Semmes in 3 provided the LittlewoodPaley theory for spaces of homogeneous type introduced by Coifman and Weiss in [2]. To be precise, if $\left\{S_{k}\right\}_{k=-\infty}^{\infty}$ is an approximation to the identity on a space of homogeneous type, then their kernels $\left\{S_{k}(x, y)\right\}_{k=1}^{\infty}$ satisfy certain size and regularity conditions; see [3] for the construction of such an approximation to the identity. It is worth pointing out that the doubling property plays an important role in this construction. Set $D_{k}=S_{k}-S_{k-1}$. Based on Coifman's ideas (see [3] for the details), at least formally, the identity operator $I$ can be written as

$$
\begin{aligned}
I & =\sum_{k=-\infty}^{\infty} D_{k} \\
& =\left(\sum_{k=-\infty}^{\infty} D_{k}\right)\left(\sum_{j=-\infty}^{\infty} D_{j}\right) \\
& =\sum_{|k-j| \leq N} D_{k} D_{j}+\sum_{|k-j|>N} D_{k} D_{j} \\
& =T_{N}+R_{N} .
\end{aligned}
$$

David, Journé and Semmes proved that if $N$ is large enough, then $R_{N}$ is bounded on $L^{p}(X), 1<p<\infty$, with the operator norm less than 1 . Therefore, if $N$ is large 
enough and $D_{k}^{N}=\sum_{|j| \leq N} D_{k+j}$ for $k \in \mathbb{Z}$, they obtained the following Calderóntype reproducing formulae:

$$
f=\sum_{k=-\infty}^{\infty} T_{N}^{-1} D_{k}^{N} D_{k}(f)=\sum_{k=-\infty}^{\infty} D_{k} D_{k}^{N} T_{N}^{-1}(f),
$$

where $T_{N}^{-1}$ is the inverse of $T_{N}$ and the series converge in $L^{p}(X), 1<p<\infty$. Using this formula, they were able to obtain the Littlewood-Paley theory for the space $L^{p}(X)$ : There exists a constant $C>0$ such that for all $f \in L^{p}(X), 1<p<\infty$,

$$
C^{-1}\|f\|_{L^{p}(X)} \leq\left\|\left\{\sum_{k=-\infty}^{\infty}\left|D_{k}(f)\right|^{2}\right\}^{1 / 2}\right\|_{L^{p}(X)} \leq C\|f\|_{L^{p}(X)} .
$$

In [12, via the Littlewood-Paley theory, Sawyer and the second author of this paper introduced the Besov space on spaces of homogeneous type. More precisely, they first introduced a space of test functions, $\mathcal{M}(X)$ (which is also called the space of smooth molecules in [9]), and approximations to the identity $\left\{S_{k}\right\}_{k=-\infty}^{\infty}$ whose kernels satisfy all size and regularity conditions (as mentioned above in Coifman's construction), and furthermore, the second difference smoothness condition. They then proved that if $N$ is large enough, $R_{N}$ is bounded on the space of test functions, $\mathcal{M}(X)$, with the operator norm less than 1 . Using this fact, they obtained the Calderón reproducing formula. To be precise, let $\left\{S_{k}\right\}_{k=-\infty}^{\infty}$ be any approximation to the identity as in [12] and $D_{k}=S_{k}-S_{k-1}$ for $k \in \mathbb{Z}$. Then there exist families of operators $\left\{\widetilde{D}_{k}\right\}_{k=-\infty}^{\infty}$ and $\left\{\bar{D}_{k}\right\}_{k=-\infty}^{\infty}$ such that

$$
f=\sum_{k=-\infty}^{\infty} \widetilde{D}_{k} D_{k}(f)=\sum_{k=-\infty}^{\infty} D_{k} \bar{D}_{k}(f)
$$

where the series converge in the norms of the space $L^{p}(X), 1<p<\infty$, the space $\mathcal{M}(X)$ and the dual space $(\mathcal{M}(X))^{\prime}$, respectively.

Note that the formula in (1.5) is similar to that in (1.2), and the second difference smoothness condition plays a crucial role in establishing (1.5). Thus, the theory of Besov spaces on spaces of homogeneous type can be developed as in the case of $\mathbb{R}^{d}$. More precisely, the Besov space on a space of homogeneous type $(X, \rho, \mu), \dot{B}_{p, q}^{s}(X)$ for $1 \leq p, q \leq \infty$ and $|s|<\theta$, where $\theta$ depends on the regularity of $S_{k}(x, y)$, is defined to be the set of all $f \in(\mathcal{M}(X))^{\prime}$ such that

$$
\|f\|_{\dot{B}_{p, q}^{s}(X)}=\left\{\sum_{k=-\infty}^{\infty} 2^{s k q}\left\|D_{k}(f)\right\|_{L^{p}(X)}^{q}\right\}^{1 / q}<\infty,
$$

where $D_{k}(f)(x)=\left\langle D_{k}(x, \cdot), f(\cdot)\right\rangle$; see [12] (also [10]) for the details. Again, using formulae (1.5), one showed that Besov spaces are independent of the choice of approximations to the identity $\left\{S_{k}\right\}_{k=-\infty}^{\infty}$, and, moreover, all other properties such as embedding, interpolation, duality, atomic decomposition and the $T 1$ theorem were obtained; see [12, 10, 11, 13, 14.

One of the main difficulties for developing a theory of Besov space with respect to some non-doubling measure $\mu$ which does not satisfy any regularity property, apart from the growth condition (1.1), is the construction of approximations to the identity. More recently, Tolsa constructed a "reasonable" approximation to the identity. To be precise, Tolsa in [27] constructed a sequence of integral operators 
$\left\{S_{k}\right\}_{k=-\infty}^{\infty}$ given by kernels $\left\{S_{k}(x, y)\right\}_{k=-\infty}^{\infty}$ defined on $\mathbb{R}^{d} \times \mathbb{R}^{d}$. Moreover, these kernels satisfy some appropriate size and regularity conditions and

$$
\int_{\mathbb{R}^{d}} S_{k}(x, y) d \mu(y)=1
$$

for all $x \in \operatorname{supp}(\mu)$ and $S_{k}(x, y)=S_{k}(y, x)$ for all $k \in \mathbb{Z}$. For each $k \in \mathbb{Z}$, set $D_{k}=S_{k}-S_{k-1}$, and then, again, based on Coifman's ideas mentioned above, and by use of the appropriate size and regularity conditions on $S_{k}(x, y)$, the Cotlar-Stein lemma (see [22]) and the Calderón-Zygmund theory associated to non-doubling measures, Tolsa proved that the Calderón-type reproducing formula in (1.4) still holds for non-doubling measures. Using this formula, Tolsa was able to establish a Littlewood-Paley theory associated to non-doubling measures. However, the size and regularity conditions of $S_{k}(x, y)$ constructed by Tolsa are not enough to obtain a Calderón reproducing formula similar to (1.5). A crucial observation of this paper is that if the norm $\|f\|_{\dot{B}_{p q}^{s}(\mu)}$ for all $L^{2}(\mu)$ functions $f$ is defined by

$$
\|f\|_{\dot{B}_{p q}^{s}(\mu)}=\left\{\sum_{k=-\infty}^{\infty} 2^{s k q}\left\|D_{k}(f)\right\|_{L^{p}(\mu)}^{q}\right\}^{1 / q}<\infty,
$$

where $\left\{D_{k}\right\}_{k=-\infty}^{\infty}$ are the same as in Tolsa's Calderón-type reproducing formula, then $R_{N}$ in (1.3) is bounded with respect to this norm and the operator norm is less than 1 if $N$ is large enough. Hence, $T_{N}^{-1}$ is bounded with respect to this norm. This observation leads us to introduce a new "space of test functions" defined by

$$
\dot{\mathcal{B}}_{p, q}^{s}(\mu)=\left\{f \in L^{2}(\mu):\|f\|_{\dot{B}_{p q}^{s}(\mu)}<\infty\right\} .
$$

We will prove that the Calderón-type reproducing formulae in (1.4) with Tolsa's approximations to the identity hold for the space of test functions, $\dot{\mathcal{B}}_{p, q}^{s}(\mu)$.

To show the Calderón-type reproducing formulae in (1.4) still hold on the "distribution (dual) space" $\left(\dot{\mathcal{B}}_{p, q}^{s}(\mu)\right)^{\prime}$, as for spaces of homogeneous type mentioned above, the second difference smoothness condition of the approximation to the identity is needed. We will show that Tolsa's construction of approximations to identity does have this property (see Lemma 2.1 (f) below) and, hence, the Calderón-type reproducing formulae in (1.4) on the "distribution space" $\left(\dot{\mathcal{B}}_{p, q}^{s}(\mu)\right)^{\prime}$ are obtained. As soon as the Calderón-type reproducing formulae on the distribution space are established, we can develop a theory of Besov spaces with non-doubling measures as in the cases of $\mathbb{R}^{d}$ and spaces of homogeneous type.

The plan of this paper is the following. In the next section, we will show that the approximation to the identity of Tolsa satisfies the second difference smoothness estimate. Also, in this section, we will prove that the operator $T_{N}^{-1}$ is bounded with respect to the norm $\|\cdot\|_{\dot{B}_{p q}^{s}(\mu)}$. To this end, we first prove that $R_{N}$ in (1.3) is bounded with respect to this norm with small operator norm; see Theorem 2.1 below. Some ideas of the proof of Theorem 2.1 are similar to the proof of the Cotlar-Stein lemma (see [22). The main result of this section is the Calderón-type reproducing formulae on the distribution space $\left(\dot{\mathcal{B}}_{p, q}^{s}(\mu)\right)^{\prime}$; see Theorem 2.2 below. In Section 3, we will introduce the Besov space $\dot{B}_{p q}^{s}(\mu)$ for $1 \leq p, q \leq \infty$ and $|s|<\theta$, where $\theta>0$ is a real number which depends on the non-doubling measure $\mu, C_{0}$, $n$ and $d$; see Definition 2.5 below. Moreover, if $\mu$ is the $d$-dimensional Lebesgue 
measure on $\mathbb{R}^{d}$, then $\theta=1 / 2$ (see Remark 2.1 below). In this section, we will also give some applications of these spaces. To be precise, we study the boundedness of the Riesz potential operators on these spaces, and using them, we prove that these spaces have lifting properties. Finally we consider their dual spaces in this section.

Throughout the paper, the letter $C$ will be used for constants that may change from one occurrence to another. Constants with subscripts, such as, $C_{0}$, do not change in different occurrences. The notation $A \sim B$ means that there is some constant $C>0$ such that $C^{-1} A \leq B \leq C A$. For any index $q \in[1, \infty]$, we denote by $q^{\prime}$ the conjugate index, namely $1 / q+1 / q^{\prime}=1$. We also denote $\mathbb{N} \cup\{0\}$ by $\mathbb{Z}_{+}$.

\section{Calderón-type Reproducing Formulae}

We use the same notation and definitions as in Tolsa 27] (also 28]), and for the reader's convenience, we recall some basic notation and definitions here; see [27, 28] for more details.

Throughout this paper, by a cube $Q$ we mean a closed cube with sides parallel to the axes and centered at some point of $\operatorname{supp}(\mu)$. Also, $\rho Q$ is the cube concentric with $Q$ whose side length is $\rho$ times the side length of $Q$.

We will assume that the constant $C_{0}$ in (1.1) has been chosen big enough so that for all cubes $Q \subset \mathbb{R}^{d}$, we have

$$
\mu(Q) \leq C_{0} \ell(Q)^{n},
$$

where $0<n \leq d$ and $\ell(Q)$ is the side length of the cube $Q$.

We first recall the definition of doubling cubes of Tolsa in [26, 27. Given $\alpha>1$ and $\beta>\alpha^{n}$, we say that the cube $Q \subset \mathbb{R}^{d}$ is $(\alpha, \beta)$-doubling if $\mu(\alpha Q) \leq \beta \mu(Q)$; see [26. 27. for the existence and some other basic properties of the doubling cubes.

For definiteness, if $\alpha$ and $\beta$ are not specified, by a doubling cube we mean a $\left(2,2^{d+1}\right)$-doubling cube in what follows.

Given cubes $Q, R \subset \mathbb{R}^{d}$, we denote by $z_{Q}$ the center of $Q$, and by $Q_{R}$ the smallest cube concentric with $Q$ containing $Q$ and $R$.

Definition 2.1. Given two cubes $Q, R \subset \mathbb{R}^{d}$, we define

$$
\delta(Q, R)=\max \left\{\int_{Q_{R} \backslash Q} \frac{1}{\left|x-z_{Q}\right|^{n}} d \mu(x), \int_{R_{Q} \backslash R} \frac{1}{\left|x-z_{R}\right|^{n}} d \mu(x)\right\} .
$$

We may treat points $x \in \operatorname{supp}(\mu)$ as if they were cubes (with $\ell(x)=0$ ). So, for $x, y \in \operatorname{supp}(\mu)$ and some cube $Q$, the notations $\delta(x, Q)$ and $\delta(x, y)$ make sense; see [28, 27] for some useful properties of $\delta(\cdot, \cdot)$.

We now recall the definition of cubes of different generations in [27, 28, see 27, 28, for more details.

Definition 2.2. We say that $x \in \operatorname{supp}(\mu)$ is a stopping point (or stopping cube) if $\delta(x, Q)<\infty$ for some cube $Q \ni x$ with $0<\ell(Q)<\infty$. We say that $\mathbb{R}^{d}$ is an initial cube if $\delta\left(Q, \mathbb{R}^{d}\right)<\infty$ for some cube $Q$ with $0<\ell(Q)<\infty$. The cubes $Q$ such that $0<\ell(Q)<\infty$ are called transit cubes.

Let $A$ be some big positive constant. In particular, we assume that $A$ is much bigger than the constants $\epsilon_{0}, \epsilon_{1}$ and $\gamma_{0}$, which appear, respectively, in Lemma 3.1, Lemma 3.2 and Lemma 3.3 of [27. Moreover, the constants $A, \epsilon_{0}, \epsilon_{1}$ and $\gamma_{0}$ depend only on $C_{0}, n$ and $d$. 
In what follows, for $\epsilon>0$ and $a, b \in \mathbb{R}$, the notation $a=b \pm \epsilon$ does not mean any precise equality but the estimate $|a-b| \leq \epsilon$.

Definition 2.3. Assume that $\mathbb{R}^{d}$ is not an initial cube. We fix some doubling cube $R_{0} \subset \mathbb{R}^{d}$. This will be our "reference" cube. For each $j \in \mathbb{N}$, we let $R_{-j}$ be some doubling cube concentric with $R_{0}$, containing $R_{0}$, and such that $\delta\left(R_{0}, R_{-j}\right)=$ $j A \pm \epsilon_{1}$ (which exists because of Lemma 3.3 of [27]). If $Q$ is a transit cube, we say that $Q$ is a cube of generation $k \in \mathbb{Z}$ if it is a doubling cube, and for some cube $R_{-j}$ containing $Q$ we have

$$
\delta\left(Q, R_{-j}\right)=(j+k) A \pm \epsilon_{1} .
$$

If $Q \equiv\{x\}$ is a stopping cube, we say that $Q$ is a cube of generation $k$ if for some cube $R_{-j}$ containing $x$ we have

$$
\delta\left(Q, R_{-j}\right) \leq(j+k) A \pm \epsilon_{1} .
$$

Definition 2.4. Assume that $\mathbb{R}^{d}$ is an initial cube. Then we choose $\mathbb{R}^{d}$ as our "reference": If $Q$ is a transit cube, we say that $Q$ is a cube of generation $k \geq 1$ if

$$
\delta\left(Q, \mathbb{R}^{d}\right)=k A \pm \epsilon_{1} .
$$

If $Q \equiv\{x\}$ is a stopping cube, we say that $Q$ is a cube of generation $k \geq 1$ if

$$
\delta\left(x, \mathbb{R}^{d}\right) \leq k A \pm \epsilon_{1} .
$$

Moreover, for all $k \leq 0$ we say that $\mathbb{R}^{d}$ is a cube of generation $k$.

In what follows, for any $x \in \operatorname{supp}(\mu)$, we denote by $Q_{x, k}$ some fixed doubling cube centered at $x$ of the $k$ th generation.

It is easily seen that if $A$ is big enough, then $\ell\left(Q_{x, k+1}\right) \leq \ell\left(Q_{x, k}\right) / 10$. Thus, $\ell\left(Q_{x, k}\right) \rightarrow 0$ as $k \rightarrow \infty$. In fact, the following more precise result was established in [28] by Tolsa; see also [27].

Lemma 2.1. If $A$ is big enough, then there exists some $\eta>0$ such that for $m \in \mathbb{N}$ and $2 Q_{x, k} \cap 2 Q_{y, k+m} \neq \emptyset$ with $x, y \in \operatorname{supp}(\mu), \ell\left(Q_{y, k+m}\right) \leq 2^{-\eta m} \ell\left(Q_{x, k}\right)$.

The constant $\eta$ appearing in Lemma 2.1 actually represents some kind of regularity of the approximation to the identity of Tolsa in 27, which is very important in establishing the theory of Besov spaces on $\mathbb{R}^{d}$.

Definition 2.5. Let $\theta$ be half of the maximum $\eta$ such that Lemma 2.1 holds.

Thus, $\theta$ depends only on $\mu, C_{0}, n$ and $d$. By the proof of Lemma 2.2 in [28], $\theta \in(0, \infty)$. Thus, the situation for spaces of non-homogeneous type is not the same as spaces of homogeneous type.

Remark 2.1. If $\mu$ is just the $d$-dimensional Lebesgue measure on $\mathbb{R}^{d}$, in this case, we can take $R_{0}$ in Definition 2.3 to be the unit cube centered at the original and $R_{-j}$ for $j \in \mathbb{N}$ to be the cube centered at the original with side length $2^{j}$. Let $\omega_{d-1}$ be the area of the unit ball in $\mathbb{R}^{d}$. Then $A=\omega_{d-1}$ in this case, and if $Q$ is a cube of generation $k$, then $\ell(Q) \sim 2^{-k}$. Thus, in this case, $\eta$ in Lemma 2.1 is equal to 1 and $\theta$ in Definition 2.5 is equal to $1 / 2$.

We now define

$$
\sigma \equiv 100 \epsilon_{0}+100 \epsilon_{1}+12^{n+1} C_{0} .
$$

We will also introduce two new constants $\alpha_{1}, \alpha_{2}>0$ such that

$$
\epsilon_{0}, \epsilon_{1}, C_{0} \ll \sigma \ll \alpha_{1} \ll \alpha_{2} \ll A .
$$


Definition 2.6. Let $y \in \operatorname{supp}(\mu)$. If $Q_{y, k}$ is a transit cube, we denote by $Q_{y, k}^{1}$, $Q_{y, k}^{2}, \hat{Q}_{y, k}^{2}$ and $Q_{y, k}^{3}$ some doubling cubes centered at $y$ containing $Q_{y, k}$ such that

$$
\begin{aligned}
& \delta\left(Q_{y, k}, Q_{y, k}^{1}\right)=\alpha_{1} \pm \epsilon_{1}, \\
& \delta\left(Q_{y, k}, Q_{y, k}^{2}\right)=\alpha_{1}+\alpha_{2} \pm \epsilon_{1}, \\
& \delta\left(Q_{y, k}, \hat{Q}_{y, k}^{2}\right)=\alpha_{1}+\alpha_{2}+\sigma \pm \epsilon_{1} \\
& \delta\left(Q_{y, k}, Q_{y, k}^{3}\right)=\alpha_{1}+\alpha_{2}+2 \sigma \pm \epsilon_{1} .
\end{aligned}
$$

Also, we denote by $\check{Q}_{y, k}^{1}$ and $\check{Q}_{y, k}^{1}$ some doubling cubes centered at $y$ and contained in $Q_{y, k-1}$ satisfying

$$
\begin{gathered}
\delta\left(\check{Q}_{y, k}^{1}, Q_{y, k-1}\right)=A-\alpha_{1}+\sigma \pm \epsilon_{1}, \\
\delta\left(\check{Q}_{y, k}^{1}, Q_{y, k-1}\right)=A-\alpha_{1}+2 \sigma \pm \epsilon_{1} .
\end{gathered}
$$

If any of the cubes $\check{Q}_{y, k}^{1}$ and $\check{Q}_{y, k}^{1}$ does not exist because $\delta\left(y, Q_{y, k}\right)$ is not big enough, then we let it be the point $y$.

If $Q_{y, k}=\mathbb{R}^{d}$, we set $Q_{y, k}^{1}=Q_{y, k}^{2}=\hat{Q}_{y, k}^{2}=Q_{y, k}^{3}=\check{Q}_{y, k}^{1}=\check{Q}_{y, k}^{1}=\mathbb{R}^{d}$.

If $Q_{y, k} \equiv\{y\}$ is a stopping cube and $Q_{y, k-1} \equiv\{y\}$ is also a stopping cube, we set $Q_{y, k}^{1}=Q_{y, k}^{2}=\hat{Q}_{y, k}^{2}=Q_{y, k}^{3}=\{y\}$. If $Q_{y, k} \equiv\{y\}$ is a stopping cube but $Q_{y, k-1}$ is not, then we choose $Q_{y, k}^{1}, Q_{y, k}^{2}, \hat{Q}_{y, k}^{2}$ and $Q_{y, k}^{3}$ so that they are contained in $Q_{y, k-1}$, centered at $y$ and

$$
\begin{aligned}
& \delta\left(Q_{y, k}^{1}, Q_{y, k-1}\right)=A-\alpha_{1} \pm \epsilon_{1} \\
& \delta\left(Q_{y, k}^{2}, Q_{y, k-1}\right)=A-\alpha_{1}-\alpha_{2} \pm \epsilon_{1} \\
& \delta\left(\hat{Q}_{y, k}^{2}, Q_{y, k-1}\right)=A-\alpha_{1}-\alpha_{2}-\sigma \pm \epsilon_{1} \\
& \delta\left(Q_{y, k}^{3}, Q_{y, k-1}\right)=A-\alpha_{1}-\alpha_{2}-2 \sigma \pm \epsilon_{1} .
\end{aligned}
$$

If any of these cubes does not exist because $\delta\left(y, Q_{y, k-1}\right)$ is not big enough, we let this cube be the point $y$.

The following lemma is established in 28]; see also [27.

Lemma 2.2. Let $y \in \operatorname{supp}(\mu)$. If we choose the constants $\alpha_{1}, \alpha_{2}$ and $A$ big enough, we have

$$
Q_{y, k} \subset \check{Q}_{y, k}^{1} \subset \check{Q}_{y, k}^{1} \subset Q_{y, k}^{1} \subset Q_{y, k}^{2} \subset \hat{Q}_{y, k}^{2} \subset Q_{y, k}^{3} \subset Q_{y, k-1} .
$$

For a fixed $k$, cubes of the $k$ th generation may have very different sizes for different $y$ 's. The same happens for the cubes $Q_{y, k}^{1}$ and $Q_{y, k}^{2}$. Nevertheless, in 28] (see also [27]), it has been shown that we still have some kind of regularity.

Lemma 2.3. Given $x, y \in \operatorname{supp}(\mu)$, let $Q_{x}$ and $Q_{y}$ be the cubes centered at $x$ and $y$ respectively, and assume that $Q_{x} \cap Q_{y} \neq \emptyset$ and that there exists some cube $R_{0}$ containing $Q_{x} \cup Q_{y}$ with $\left|\delta\left(Q_{x}, R_{0}\right)-\delta\left(Q_{y}, R_{0}\right)\right| \leq 10 \epsilon_{1}$. If $R_{y}$ is some cube centered at $y$ containing $Q_{y}$ with $\delta\left(Q_{y}, R_{y}\right) \geq \sigma-10 \epsilon_{1}$, then $Q_{x} \subset R_{y}$. As a consequence, we have if $Q_{x, k} \cap Q_{y, k} \neq \emptyset$, then $Q_{x, k} \subset Q_{y, k-1}$.

To recall the construction of the approximation to the identity of Tolsa in [27, we first recall the following auxiliary functions $\psi_{y, k}$ and $\varphi_{x, k}$. 
Definition 2.7. For any $y \in \operatorname{supp}(\mu)$, the function $\psi_{y, k}$ is a function on $\mathbb{R}^{d}$ such that

$$
\begin{aligned}
& \text { (a) } \quad 0 \leq \psi_{y, k}(x) \leq \min \left(\frac{4}{\ell\left(Q_{y, k}^{1}\right)^{n}}, \frac{1}{|y-x|^{n}}\right), \\
& \text { (b) } \psi_{y, k}(x)=\frac{1}{|x-y|^{n}} \text { if } x \in \hat{Q}_{y, k}^{2} \backslash Q_{y, k}^{1}, \\
& \text { (c) } \quad \operatorname{supp}\left(\psi_{y, k}\right) \subset Q_{y, k}^{3}, \\
& \text { (d) }\left|\psi_{y, k}^{\prime}(x)\right| \leq C \min \left(\frac{1}{\ell\left(Q_{y, k}^{1}\right)^{n+1}}, \frac{1}{|y-x|^{n+1}}\right),
\end{aligned}
$$

where $C>0$ is large enough.

Choosing $\alpha_{2}>0$ big enough, for all $y \in \operatorname{supp}(\mu)$, we define $\varphi_{y, k}(x)=\alpha_{2}^{-1} \psi_{y, k}(x)$.

Using $\left\{\varphi_{y, k}\right\}$, we can recall the definition of the approximation to the identity $\left\{S_{k}\right\}_{k \in \mathbb{Z}}$ in $[27$.

Definition 2.8. Let $f \in L_{\mathrm{loc}}^{1}(\mu)$ and $x \in \operatorname{supp}(\mu)$. If $Q_{x, k} \neq \mathbb{R}^{d}$, then we set

$$
\widetilde{S}_{k} f(x)=\int_{\mathbb{R}^{d}} \varphi_{y, k}(x) f(y) d \mu(y)+\max \left(0, \frac{1}{4}-\int_{\mathbb{R}^{d}} \varphi_{y, k}(x) d \mu(y)\right) f(x) .
$$

Assume that $Q_{x, k} \neq \mathbb{R}^{d}$ for some $x \in \operatorname{supp}(\mu)$. Let $M_{k}$ be the operator of multiplication by $M_{k}(x) \equiv 1 / \widetilde{S}_{k} 1(x)$ and let $W_{k}$ be the operator of multiplication by

$$
W_{k}(x) \equiv 1 / \widetilde{S}_{k}^{*}\left(1 / \widetilde{S}_{k} 1\right)(x) .
$$

We set $S_{k} \equiv M_{k} \widetilde{S}_{k} W_{k} \widetilde{S}_{k}^{*} M_{k}$. If $Q_{x, k}=\mathbb{R}^{d}$ for some $x \in \operatorname{supp}(\mu)$, then we set $S_{k} \equiv 0$.

Observe that, formally, $\widetilde{S}_{k}$ is an integral operator with the following positive kernel:

$$
\widetilde{S}_{k}(x, y)=\varphi_{y, k}(x)+\max \left(0, \frac{1}{4}-\int_{\mathbb{R}^{d}} \varphi_{y, k}(x) d \mu(y)\right) d z_{x}(y),
$$

where $\delta_{x}$ is the Dirac delta at $x$, and that if $Q_{x, k}$ and $Q_{y, k}$ are transit cubes, then $S_{k}$ is also an integral operator with the following positive kernel:

$$
S_{k}(x, y)=\int_{\mathbb{R}^{d}} M_{k}(x) \widetilde{S}_{k}(x, z) W_{k}(z) \widetilde{S}_{k}(y, z) M_{k}(y) d \mu(z) .
$$

We now recall some basic properties of the kernels $\left\{S_{k}(x, y)\right\}_{k \in \mathbb{Z}}$ in (2.2), moreover, we will verify that they also have the regularity of the second difference.

Lemma 2.4. There exist a sequence of operators of $\left\{S_{k}\right\}_{k=-\infty}^{\infty}$ with kernels $S_{k}(x, y)$ defined on $\mathbb{R}^{d} \times \mathbb{R}^{d}$. For each $k \in \mathbb{Z}$ the following properties hold:

(a) $S_{k}(x, y)=S_{k}(y, x)$.

(b) $\int_{\mathbb{R}^{d}} S_{k}(x, y) d \mu(y)=1$ for $x \in \operatorname{supp}(\mu)$.

(c) If $Q_{x, k}$ is a transit cube, then $\operatorname{supp}\left(S_{k}(x, \cdot)\right) \subset Q_{x, k-1}$.

(d) If $Q_{x, k}$ and $Q_{y, k}$ are transit cubes, then

$$
0 \leq S_{k}(x, y) \leq \frac{C}{\left(\ell\left(Q_{x, k}\right)+\ell\left(Q_{y, k}\right)+|x-y|\right)^{n}} .
$$


(e) If $Q_{x, k}, Q_{x^{\prime}, k}, Q_{y, k}$ are transit cubes, and $x, x^{\prime} \in Q_{x_{0}, k}$ for some $x_{0} \in$ $\operatorname{supp}(\mu)$, then

$$
\left|S_{k}(x, y)-S_{k}\left(x^{\prime}, y\right)\right| \leq C \frac{\left|x-x^{\prime}\right|}{\ell\left(Q_{x_{0}, k}\right)} \frac{1}{\left(\ell\left(Q_{x, k}\right)+\ell\left(Q_{y, k}\right)+|x-y|\right)^{n}} .
$$

(f) If $Q_{x, k}, Q_{x^{\prime}, k}, Q_{y, k}$ and $Q_{y^{\prime}, k}$ are transit cubes, $x, x^{\prime} \in Q_{x_{0}, k}$ and $y, y^{\prime} \in$ $Q_{y_{0}, k}$ for some $x_{0}, y_{0} \in \operatorname{supp}(\mu)$, then

$$
\begin{aligned}
& \left|\left[S_{k}(x, y)-S_{k}\left(x^{\prime}, y\right)\right]-\left[S_{k}\left(x, y^{\prime}\right)-S_{k}\left(x^{\prime}, y^{\prime}\right)\right]\right| \\
& \leq C \frac{\left|x-x^{\prime}\right|}{\ell\left(Q_{x_{0}, k}\right)} \frac{\left|y-y^{\prime}\right|}{\ell\left(Q_{y_{0}, k}\right)} \frac{1}{\left(\ell\left(Q_{x, k}\right)+\ell\left(Q_{y, k}\right)+|x-y|\right)^{n}} .
\end{aligned}
$$

Proof. Let $S_{k}(x, y)$ for $k \in \mathbb{Z}$ be the same as in (2.2). Tolsa in 27] has proved that $S_{k}(x, y)$ satisfy (a), (b), (c), (d) and (e); see 27] for the details. We only need to verify that Tolsa's approximations to the identity satisfy the second difference smoothness condition in (2.3).

Let $Q_{x, k}, Q_{x^{\prime}, k}$ and $Q_{y, k}$ be the transit cubes as in Definition 2.2. To verify (2.3), by (2.2), we have

$$
\begin{aligned}
& {\left[S_{k}(x, y)-S_{k}\left(x^{\prime}, y\right)\right]-\left[S_{k}\left(x, y^{\prime}\right)-S_{k}\left(x^{\prime}, y^{\prime}\right)\right]} \\
& \quad=\int_{\mathbb{R}^{d}}\left[M_{k}(x) \widetilde{S}_{k}(x, z)-M_{k}\left(x^{\prime}\right) \widetilde{S}_{k}\left(x^{\prime}, z\right)\right] W_{k}(z) \\
& \quad \times\left[\widetilde{S}_{k}(y, z) M_{k}(y)-\widetilde{S}_{k}\left(y^{\prime}, z\right) M_{k}\left(y^{\prime}\right)\right] d \mu(z) \\
& \quad=\left[M_{k}(x)-M_{k}\left(x^{\prime}\right)\right] M_{k}(y)\left\{\int_{\mathbb{R}^{d}} \widetilde{S}_{k}(x, z) W_{k}(z)\left[\widetilde{S}_{k}(y, z)-\widetilde{S}_{k}\left(y^{\prime}, z\right)\right] d \mu(z)\right\} \\
& \quad+\left[M_{k}(x)-M_{k}\left(x^{\prime}\right)\right]\left[M_{k}(y)-M_{k}\left(y^{\prime}\right)\right]\left\{\int_{\mathbb{R}^{d}} \widetilde{S}_{k}(x, z) W_{k}(z) \widetilde{S}_{k}\left(y^{\prime}, z\right) d \mu(z)\right\} \\
& \quad+M_{k}\left(x^{\prime}\right)\left[M_{k}(y)-M_{k}\left(y^{\prime}\right)\right]\left\{\int_{\mathbb{R}^{d}}\left[\widetilde{S}_{k}(x, z)-\widetilde{S}_{k}\left(x^{\prime}, z\right)\right] W_{k}(z) \widetilde{S}_{k}\left(y^{\prime}, z\right) d \mu(z)\right\} \\
& \quad+M_{k}\left(x^{\prime}\right) M_{k}(y)\left\{\int_{\mathbb{R}^{d}}\left[\widetilde{S}_{k}(x, z)-\widetilde{S}_{k}\left(x^{\prime}, z\right)\right] W_{k}(z)\left[\widetilde{S}_{k}(y, z)-\widetilde{S}_{k}\left(y^{\prime}, z\right)\right] d \mu(z)\right\} \\
& \quad=B_{1}+B_{2}+B_{3}+B_{4} .
\end{aligned}
$$

It was proved by Tolsa in [27, pp. 78-79] that for $x, x^{\prime} \in Q_{x_{0}, k}$,

$$
\left|M_{k}(x)-M_{k}\left(x^{\prime}\right)\right| \leq C \frac{\left|x-x^{\prime}\right|}{\ell\left(Q_{x_{0}, k}\right)}
$$

and

$$
\begin{aligned}
& \int_{\mathbb{R}^{d}}\left|\widetilde{S}_{k}(y, z)-\widetilde{S}_{k}\left(y^{\prime}, z\right)\right| \widetilde{S}_{k}(x, z) d \mu(z) \\
& \leq C \frac{\left|y-y^{\prime}\right|}{\ell\left(Q_{y_{0}, k}\right)} \frac{1}{\left(\ell\left(Q_{x, k}\right)+\ell\left(Q_{y, k}\right)+|x-y|\right)^{n}} .
\end{aligned}
$$

From the fact that for $z \in \operatorname{supp}(\mu)$ satisfying $Q_{z, k} \neq \mathbb{R}^{d}$,

$$
0 \leq W_{k}(z) \leq 6 \text { and } 2 / 3 \leq M_{k}(z) \leq 4
$$

(see Lemma 5.1 in [27), the estimates (2.4) and (2.5), it follows that

$$
\left|B_{1}\right| \leq C \frac{\left|x-x^{\prime}\right|}{\ell\left(Q_{x_{0}, k}\right)} \frac{\left|y-y^{\prime}\right|}{\ell\left(Q_{y_{0}, k}\right)} \frac{1}{\left(\ell\left(Q_{x, k}\right)+\ell\left(Q_{y, k}\right)+|x-y|\right)^{n}},
$$

which is a desired estimate. 
To estimate $B_{2}$, we first verify that

$$
\int_{\mathbb{R}^{d}} \widetilde{S}_{k}(x, z) \widetilde{S}_{k}\left(y^{\prime}, z\right) d \mu(z) \leq \frac{C}{\left(\ell\left(Q_{x, k}\right)+\ell\left(Q_{y, k}\right)+|x-y|\right)^{n}} .
$$

By Lemma 4.4(b) and (c) in [27] and noting that $Q_{x, k} \subset \check{Q}_{x, k}^{1}$ (see Lemma 2.2), we have

$$
0 \leq \widetilde{S}_{k}(x, z)=\varphi_{z, k}(x) \leq \frac{C}{\ell\left(\check{Q}_{x, k}^{1}\right)^{n}} \leq \frac{C}{\ell\left(Q_{x, k}\right)^{n}} .
$$

On the other hand, from Lemma 2.3 , it is easy to deduce that $y \in Q_{y_{0}, k}$ implies that $Q_{y, k} \subset \check{Q}_{y_{0}, k}^{1}$. Moreover, the fact that $Q_{y^{\prime}, k} \subset \check{Q}_{y^{\prime}, k}^{1}$ and $y^{\prime} \in Q_{y_{0}, k} \subset \check{Q}_{y_{0}, k}^{1}$ imply that $\check{Q}_{y^{\prime}, k}^{1} \cap \check{Q}_{y_{0}, k}^{1} \neq \emptyset$, which together with Lemma 2.3 further indicates that $\check{Q}_{y_{0}, k}^{1} \subset \check{Q}_{y^{\prime}, k}^{1}$. Thus,

$$
Q_{y_{0}, k} \cup Q_{y, k} \subset \check{Q}_{y^{\prime}, k}^{1} .
$$

The fact (2.10) and the estimate (2.9) yield

$$
0 \leq \widetilde{S}_{k}\left(y^{\prime}, z\right)=\varphi_{z, k}\left(y^{\prime}\right) \leq \frac{C}{\ell\left(\check{Q}_{y^{\prime}, k}^{1}\right)^{n}} \leq \frac{C}{\ell\left(Q_{y, k}\right)^{n}} .
$$

The estimates (2.9) and (2.11), together with the fact that for all $x \in \operatorname{supp}(\mu)$,

$$
\int_{\mathbb{R}^{d}} \widetilde{S}_{k}(x, z) d \mu(z) \leq C
$$

(see Lemma 4.5 in [27]), tell us that

$$
\int_{\mathbb{R}^{d}} \widetilde{S}_{k}(x, z) \widetilde{S}_{k}\left(y^{\prime}, z\right) d \mu(z) \leq C \min \left\{\frac{1}{\ell\left(Q_{x, k}\right)^{n}}, \frac{1}{\ell\left(Q_{y, k}\right)^{n}}\right\} .
$$

Lemma 4.4(b) and (c) in [27] also imply that

$$
\widetilde{S}_{k}(x, z) \leq \frac{C}{|x-z|^{n}} \quad \text { and } \quad \widetilde{S}_{k}\left(y^{\prime}, z\right)=\varphi_{z, k}\left(y^{\prime}\right) \leq \frac{C}{\left(\ell\left(\check{Q}_{y^{\prime}, k}^{1}\right)+\left|y^{\prime}-z\right|\right)^{n}}
$$

which, together with (2.12), further yields

$$
\begin{aligned}
& \int_{\mathbb{R}^{d}} \widetilde{S}_{k}(x, z) \widetilde{S}_{k}\left(y^{\prime}, z\right) d \mu(z) \\
& \leq C \int_{|x-z| \geq|x-y| / 2} \frac{1}{|x-z|^{n}} \widetilde{S}_{k}\left(y^{\prime}, z\right) d \mu(z) \\
& \quad+C \int_{|x-z|<|x-y| / 2} \widetilde{S}_{k}(x, z) \frac{1}{\left(\ell\left(\check{Q}_{y^{\prime}, k}^{1}\right)+\left|y^{\prime}-z\right|\right)^{n}} d \mu(z) \\
& \leq \frac{C}{|x-y|^{n}} \int_{|x-z|<|x-y| / 2} \widetilde{S}_{k}(x, z) \frac{1}{\left(\ell\left(Q_{y, k}\right)+|y-z|\right)^{n}} d \mu(z) \\
& \leq \frac{C}{|x-y|^{n}},
\end{aligned}
$$

where we used the fact that

$$
\ell\left(Q_{y, k}\right)+|y-z| \leq C\left(\ell\left(\check{Q}_{y^{\prime}, k}^{1}\right)+\left|y^{\prime}-z\right|\right) .
$$

This can be deduced from (2.10) and

$$
|y-z| \leq\left|y-y^{\prime}\right|+\left|y^{\prime}-z\right| \leq \sqrt{d} \ell\left(Q_{y_{0}, k}\right)+\left|y^{\prime}-z\right| \leq \sqrt{d}\left(\ell\left(\check{Q}_{y^{\prime}, k}^{1}\right)+\left|y^{\prime}-z\right|\right),
$$


since $y, y^{\prime} \in Q_{y_{0}, k}$. The estimates (2.13) and (2.15) show (2.8). The estimates (2.4), (2.6) and (2.8) yield

$$
\left|B_{2}\right| \leq C \frac{\left|x-x^{\prime}\right|}{\ell\left(Q_{x_{0}, k}\right)} \frac{\left|y-y^{\prime}\right|}{\ell\left(Q_{y_{0}, k}\right)} \frac{1}{\left(\ell\left(Q_{x, k}\right)+\ell\left(Q_{y, k}\right)+|x-y|\right)^{n}},
$$

which is a desired estimate.

To estimate $B_{3}$, noting that for all $w \in Q_{x_{0}, k}$,

$$
\left|\varphi_{z, k}^{\prime}(w)\right| \leq \frac{C}{\left(\ell\left(\check{Q}_{x_{0}, k}^{1}\right)+|w-z|\right)^{n+1}}
$$

(see (33) in [27]), we obtain

$$
\begin{aligned}
& \int_{\mathbb{R}^{d}}\left|\widetilde{S}_{k}(x, z)-\widetilde{S}_{k}\left(x^{\prime}, z\right)\right| \widetilde{S}_{k}\left(y^{\prime}, z\right) d \mu(z) \\
& \leq C \int_{\mathbb{R}^{d}} \frac{\left|x-x^{\prime}\right|}{\left(\ell\left(\check{Q}_{x_{0}, k}^{1}\right)+|x-z|\right)^{n+1}} \widetilde{S}_{k}\left(y^{\prime}, z\right) d \mu(z) \\
& \leq C \int_{|z-y| \geq|x-y| / 2} \frac{\left|x-x^{\prime}\right|}{\left(\ell\left(\check{Q}_{x_{0}, k}^{1}\right)+|x-z|\right)^{n+1}} \widetilde{S}_{k}\left(y^{\prime}, z\right) d \mu(z) \\
& \quad+C \int_{|z-y|<|x-y| / 2} \frac{\left|x-x^{\prime}\right|}{\left(\ell\left(\check{Q}_{x_{0}, k}^{1}\right)+|x-z|\right)^{n+1}} \widetilde{S}_{k}\left(y^{\prime}, z\right) d \mu(z) \\
& =I_{1}+I_{2} .
\end{aligned}
$$

For $I_{1}$, from (2.14) and (2.16), it follows that

$$
\begin{aligned}
I_{1} & \leq C \int_{|z-y| \geq|x-y| / 2} \frac{\left|x-x^{\prime}\right|}{\left(\ell\left(\check{Q}_{x_{0}, k}^{1}\right)+|x-z|\right)^{n+1}} \frac{1}{\left(\ell\left(\check{Q}_{y^{\prime}, k}^{1}\right)+\left|y^{\prime}-z\right|\right)^{n}} d \mu(z) \\
& \leq C \int_{|z-y| \geq|x-y| / 2} \frac{\left|x-x^{\prime}\right|}{\left(\ell\left(\check{Q}_{x_{0}, k}^{1}\right)+|x-z|\right)^{n+1}} \frac{1}{\left(\ell\left(Q_{y, k}\right)+|y-z|\right)^{n}} d \mu(z) \\
& \leq C \frac{1}{\left(\ell\left(Q_{y, k}\right)+|x-y|\right)^{n}} \int_{\mathbb{R}^{d}} \frac{\left|x-x^{\prime}\right|}{\left(\ell\left(\check{Q}_{x_{0}, k}^{1}\right)+|x-z|\right)^{n+1}} d \mu(z) \\
& \leq C \frac{\left|x-x^{\prime}\right|}{\ell\left(Q_{x_{0}, k}\right)} \frac{1}{\left(\ell\left(Q_{y, k}\right)+|x-y|\right)^{n}} .
\end{aligned}
$$

On the other hand, $x \in Q_{x_{0}, k}$ and Lemma 2.3 lead to $Q_{x, k} \subset \check{Q}_{x_{0}, k}^{1}$. This fact, $Q_{x_{0}, k} \subset \check{Q}_{x_{0}, k}^{1}$ and (2.12) imply that

$$
\begin{aligned}
I_{1} & \leq C \frac{\left|x-x^{\prime}\right|}{\ell\left(Q_{x_{0}, k}\right)} \frac{1}{\ell\left(Q_{x, k}\right)^{n}} \int_{\mathbb{R}^{d}} \widetilde{S}_{k}\left(y^{\prime}, z\right) d \mu(z) \\
& \leq C \frac{\left|x-x^{\prime}\right|}{\ell\left(Q_{x_{0}, k}\right)} \frac{1}{\ell\left(Q_{x, k}\right)^{n}} .
\end{aligned}
$$

The estimates (2.19) and (2.20) yield

$$
I_{1} \leq C \frac{\left|x-x^{\prime}\right|}{\ell\left(Q_{x_{0}, k}\right)} \frac{1}{\left(\ell\left(Q_{x, k}\right)+\ell\left(Q_{y, k}\right)+|x-y|\right)^{n}} .
$$


For $I_{2}$, noting that it was proved by Tolsa in [27, p. 79] that

$$
\ell\left(Q_{x, k}\right)+\ell\left(Q_{y, k}\right) \leq C\left(\ell\left(\check{Q}_{x_{0}, k}^{1}\right)+|x-y|\right),
$$

by $(2.12)$, we then have

$$
\begin{aligned}
I_{2} & \leq C \int_{|z-y|<|x-y| / 2} \frac{\left|x-x^{\prime}\right|}{\left(\ell\left(\check{Q}_{x_{0}, k}^{1}\right)+|x-z|\right)^{n+1}} \widetilde{S}_{k}\left(y^{\prime}, z\right) d \mu(z) \\
& \leq C \frac{\left|x-x^{\prime}\right|}{\ell\left(Q_{x_{0}, k}\right)} \frac{1}{\left(\ell\left(\check{Q}_{x_{0}, k}^{1}\right)+|x-y|\right)^{n}} \\
& \leq C \frac{\left|x-x^{\prime}\right|}{\ell\left(Q_{x_{0}, k}\right)} \frac{1}{\left(\ell\left(Q_{x, k}\right)+\ell\left(Q_{y, k}\right)+|x-y|\right)^{n}} .
\end{aligned}
$$

Combining (2.21), (2.23), (2.4) and (2.6) yields

$$
\left|B_{3}\right| \leq C \frac{\left|x-x^{\prime}\right|}{\ell\left(Q_{x_{0}, k}\right)} \frac{\left|y-y^{\prime}\right|}{\ell\left(Q_{y_{0}, k}\right)} \frac{1}{\left(\ell\left(Q_{x, k}\right)+\ell\left(Q_{y, k}\right)+|x-y|\right)^{n}} .
$$

Finally, by (2.18) and (2.6), we have

$$
\begin{aligned}
\left|B_{4}\right| \leq & C \int_{\mathbb{R}^{d}} \frac{\left|x-x^{\prime}\right|}{\left(\ell\left(\check{Q}_{x_{0}, k}^{1}\right)+|x-z|\right)^{n+1}} \frac{\left|y-y^{\prime}\right|}{\left(\ell\left(\check{Q}_{y_{0}, k}^{1}\right)+|y-z|\right)^{n+1}} d \mu(z) \\
= & C \int_{|y-z| \geq|x-y| / 2} \frac{\left|x-x^{\prime}\right|}{\left(\ell\left(\check{Q}_{x_{0}, k}^{1}\right)+|x-z|\right)^{n+1}} \frac{\left|y-y^{\prime}\right|}{\left(\ell\left(\check{Q}_{y_{0}, k}^{1}\right)+|y-z|\right)^{n+1}} d \mu(z) \\
& +C \int_{|y-z|<|x-y| / 2} \frac{\left|x-x^{\prime}\right|}{\left(\ell\left(\check{Q}_{x_{0}, k}^{1}\right)+|x-z|\right)^{n+1}} \frac{\left|y-y^{\prime}\right|}{\left(\ell\left(\check{Q}_{y_{0}, k}^{1}\right)+|y-z|\right)^{n+1}} d \mu(z) \\
= & B_{4}^{1}+B_{4}^{2} .
\end{aligned}
$$

An easy computation implies that

$$
\begin{aligned}
B_{4}^{1} & \leq C \frac{\left|y-y^{\prime}\right|}{\left(\ell\left(\check{Q}_{y_{0}, k}^{1}\right)+|x-y|\right)^{n+1}} \int_{\mathbb{R}^{d}} \frac{\left|x-x^{\prime}\right|}{\left(\ell\left(\check{Q}_{x_{0}, k}^{1}\right)+|x-z|\right)^{n+1}} d \mu(z) \\
& \leq C \frac{\left|x-x^{\prime}\right|}{\ell\left(Q_{x_{0}, k}\right)} \frac{\left|y-y^{\prime}\right|}{\ell\left(Q_{y_{0}, k}\right)} \frac{1}{\left(\ell\left(\check{Q}_{y_{0}, k}^{1}\right)+|x-y|\right)^{n}} .
\end{aligned}
$$

Since $y \in Q_{y_{0}, k}$, then $Q_{y, k} \cap Q_{y_{0}, k} \neq \emptyset$ which, by Lemma 2.3, implies that

$$
Q_{y, k} \subset \check{Q}_{y_{0}, k}^{1} \subset \check{Q}_{y_{0}, k}^{1} \text {. }
$$

Thus,

$$
\ell\left(Q_{y, k}\right) \leq \ell\left(\check{Q}_{y_{0}, k}^{1}\right)
$$

Therefore,

$$
\ell\left(Q_{y, k}\right)+|x-y| \leq C\left(\ell\left(\check{Q}_{y_{0}, k}^{1}\right)+|x-y|\right) .
$$

If $|x-y| \leq \ell\left(Q_{x, k}\right) / 2$, then $y \in Q_{x, k}$ and $Q_{x, k} \cap Q_{y, k} \neq \emptyset$, and hence, $Q_{x, k} \cap \check{Q}_{y_{0}, k}^{1} \neq$ $\emptyset$. By Lemma 2.3 again, we obtain $Q_{x, k} \subset \check{Q}_{y_{0}, k}^{1}$. That is, $\ell\left(Q_{x, k}\right) \leq \ell\left(\check{Q}_{y_{0}, k}^{1}\right)$. From this, it follows that

$$
\ell\left(Q_{x, k}\right)+\ell\left(Q_{y, k}\right)+|x-y| \leq C\left(\ell\left(\check{Q}_{y_{0}, k}^{1}\right)+|x-y|\right) .
$$


By (2.25), we can easily see that this is also true if $|x-y|>\ell\left(Q_{x, k}\right) / 2$. Thus, we always have

$$
B_{4}^{1} \leq C \frac{\left|x-x^{\prime}\right|}{\ell\left(Q_{x_{0}, k}\right)} \frac{\left|y-y^{\prime}\right|}{\ell\left(Q_{y_{0}, k}\right)} \frac{1}{\left(\ell\left(Q_{x, k}\right)+\ell\left(Q_{y, k}\right)+|x-y|\right)^{n}},
$$

which is a desired estimate. We now estimate $B_{4}^{2}$. Again an easy computation, $(2.25)$ and (2.22) indicate that

$$
\begin{aligned}
B_{4}^{2} & \leq C \frac{\left|x-x^{\prime}\right|}{\left(\ell\left(\check{Q}_{x_{0}, k}^{1}\right)+|x-y|\right)^{n+1}} \int_{\mathbb{R}^{d}} \frac{\left|y-y^{\prime}\right|}{\left(\ell\left(\check{Q}_{y_{0}, k}^{1}\right)+|y-z|\right)^{n+1}} d \mu(z) \\
& \leq C \frac{\left|x-x^{\prime}\right|}{\left(\ell\left(\check{Q}_{x_{0}, k}^{1}\right)+|x-y|\right)^{n+1}} \frac{\left|y-y^{\prime}\right|}{\ell\left(\check{Q}_{y_{0}, k}^{1}\right)} \\
& \leq C \frac{\left|x-x^{\prime}\right|}{\ell\left(Q_{x_{0}, k}\right)} \frac{\left|y-y^{\prime}\right|}{\ell\left(Q_{y_{0}, k}\right)} \frac{1}{\left(\ell\left(\check{Q}_{x_{0}, k}^{1}\right)+|x-y|\right)^{n}} \\
& \leq C \frac{\left|x-x^{\prime}\right|}{\ell\left(Q_{x_{0}, k}\right)} \frac{\left|y-y^{\prime}\right|}{\ell\left(Q_{y_{0}, k}\right)} \frac{1}{\left(\ell\left(Q_{x, k}\right)+\ell\left(Q_{y, k}\right)+|x-y|\right)^{n}} .
\end{aligned}
$$

Combining the estimates (2.26) and (2.27), we obtain

$$
\left|B_{4}\right| \leq C \frac{\left|x-x^{\prime}\right|}{\ell\left(Q_{x_{0}, k}\right)} \frac{\left|y-y^{\prime}\right|}{\ell\left(Q_{y_{0}, k}\right)} \frac{1}{\left(\ell\left(Q_{x, k}\right)+\ell\left(Q_{y, k}\right)+|x-y|\right)^{n}} .
$$

Now (2.3) follows from the estimates (2.7), (2.17), (2.24) and (2.28), and hence, we have completed the proof of Lemma 2.4.

Remark 2.2. Taking the (formal) Definition 2.8 of the kernels $\widetilde{S}_{k}(x, y)$, it is easily seen that the properties of the kernels $S_{k}(x, y)$ in (a), (b), (c), (d), (e) and (f) of Lemma 2.4 also hold without assuming that $Q_{x, k}, Q_{x^{\prime}, k}$ and $Q_{y, k}$ are transit cubes; see also [27. In what follows, without loss of generality, we will always assume that $Q_{x, k}, Q_{x^{\prime}, k}$ and $Q_{y, k}$ are transit cubes.

Definition 2.9. A sequence of operators $\left\{S_{k}\right\}_{k \in \mathbb{Z}}$ is said to be an approximation to the identity associated to non-doubling measure $\mu$ if $\left\{S_{k}(x, y)\right\}_{k=-\infty}^{\infty}$, the kernels of $\left\{S_{k}\right\}_{k=-\infty}^{\infty}$, satisfy conditions (a), (b), (c), (d), (e) and (f) of Lemma 2.4

Now, let $\left\{S_{k}\right\}_{k \in \mathbb{Z}}$ be an approximation to the identity as in Definition 2.9 and set $D_{k}=S_{k}-S_{k-1}$ for $k \in \mathbb{Z}$. Following [3] and [27, based on Coifman's idea, we can write

$$
I=T_{N}+R_{N},
$$

where $T_{N}=\sum_{|k-j| \leq N} D_{k} D_{j} \quad$ and $\quad R_{N}=\sum_{|k-j|>N} D_{k} D_{j}$; see also (1.3).

If we set $D_{k}^{N}=\sum_{|j| \leq N} D_{k+j}$ for $k \in \mathbb{Z}$, then we can also write

$$
T_{N}=\sum_{k \in \mathbb{Z}} D_{k}^{N} D_{k}
$$

As mentioned in the Introduction, the following result is a crucial observation of this paper. 
Theorem 2.1. Let $\left\{S_{k}\right\}_{k \in \mathbb{Z}},\left\{A_{k}\right\}_{k \in \mathbb{Z}}$ and $\left\{P_{k}\right\}_{k \in \mathbb{Z}}$ be approximations to the identity as in Definition 2.9. Set $D_{k}=S_{k}-S_{k-1}, G_{k}=A_{k}-A_{k-1}$ and $E_{k}=P_{k}-P_{k-1}$ for $k \in \mathbb{Z}$. Then, if $1 \leq p, q \leq \infty$ and $|s|<\theta$, where $\theta$ is the same as in Definition 2.5, for all $f \in L^{2}(\mu)$,

$$
\begin{aligned}
& \left\{\sum_{j=-\infty}^{\infty} 2^{j s q}\left\|E_{j} R_{N} f\right\|_{L^{p}(\mu)}^{q}\right\}^{1 / q} \\
& \leq C_{1}\left(2^{-N(s+2 \nu \theta)}+2^{-N(2 \nu \theta-s)}\right)\left\{\sum_{k=-\infty}^{\infty} 2^{k s q}\left\|G_{k} f\right\|_{L^{p}(\mu)}^{q}\right\}^{1 / q}
\end{aligned}
$$

with $C_{1}$ independent of $N$ and $f$, and $\nu \in(0,1 / 2)$ with $|s|<2 \nu \theta$. Moreover, if we choose $N \in \mathbb{N}$ such that

$$
C_{1}\left(2^{-N(s+2 \nu \theta)}+2^{-N(2 \nu \theta-s)}\right)<1,
$$

then for all $f \in L^{2}(\mu)$,

$$
\left\{\sum_{j=-\infty}^{\infty} 2^{j s q}\left\|E_{j} T_{N}^{-1} f\right\|_{L^{p}(\mu)}^{q}\right\}^{1 / q} \leq C\left\{\sum_{k=-\infty}^{\infty} 2^{k s q}\left\|G_{k} f\right\|_{L^{p}(\mu)}^{q}\right\}^{1 / q},
$$

where $C$ is independent of $f$, and $T_{N}$ and $R_{N}$ are the same as in (2.29).

To show Theorem 2.1, we need the following lemma.

Lemma 2.5. Let $\theta$ be the same as in Definition 2.5 and let $\left\{S_{k}\right\}_{k \in \mathbb{Z}}$ and $\left\{P_{k}\right\}_{k \in \mathbb{Z}}$ be two approximations to the identity as in Definition 2.9. Set $D_{k}=S_{k}-S_{k-1}$ and $E_{k}=P_{k}-P_{k-1}$ for $k \in \mathbb{Z}$. Then,

(i) $\operatorname{supp}\left(E_{j} D_{k}\right)(x, \cdot) \subset Q_{x, \min (j, k)-3}$ and $\operatorname{supp}\left(E_{j} D_{k}\right)(\cdot, y) \subset Q_{y, \min (j, k)-3}$ for all $j, k \in \mathbb{Z}$ and all $x, y \in \operatorname{supp}(\mu)$;

(ii) for all $x, y \in \operatorname{supp}(\mu)$ and all $j, k \in \mathbb{Z}$,

$$
\left|\left(E_{j} D_{k}\right)(x, y)\right| \leq C 2^{-2|j-k| \theta} \frac{1}{\left(\ell\left(Q_{x, \min (j, k)+1}\right)+\ell\left(Q_{y, \min (j, k)+1}\right)+|x-y|\right)^{n}} ;
$$

(iii) for $p \in[1, \infty]$ and $j, k \in \mathbb{Z}$,

$$
\left\|E_{j} D_{k}\right\|_{L^{p}(\mu) \rightarrow L^{p}(\mu)} \leq C_{2} 2^{-2|j-k| \theta},
$$

where $C_{2}>0$ is a constant depends on $p$, but not on $j$ and $k$.

Proof. The proof of this lemma is essentially contained in the proof of Lemma 6.1 in [27. For the reader's convenience, we give some details.

We first remark that one can deduce (iii) from (ii) and (i); see 27]. Thus, it suffices to verify (i) and (ii). Without loss of generality, we may assume that all the cubes $Q_{x, k}, x \in \operatorname{supp}(\mu)$ and $k \in \mathbb{Z}$, are transit cubes; see [27, pp. 80-81]. We now prove (i) and (ii) by considering several cases.

Case 1. $j \geq k+2$. In this case, completely similar to the proof of (34) in [27], we have

$$
\left|\left(E_{j} D_{k}\right)(x, y)\right| \leq C 2^{-2|j-k| \theta} \frac{1}{\left(\ell\left(Q_{x, k}\right)+\ell\left(Q_{y, k}\right)+|x-y|\right)^{n}} .
$$

Lemma 2.4 and Lemma 2.3 also tell us that $\operatorname{supp}\left(E_{j} D_{k}\right)(x, \cdot) \subset Q_{x, k-3}$ and

$$
\operatorname{supp}\left(E_{j} D_{k}\right)(\cdot, y) \subset Q_{y, k-3} \text {. }
$$


Case 2. $k-1 \leq j \leq k$. In this case, it suffices to prove that

$$
\left|\left(E_{j} D_{k}\right)(x, y)\right| \leq \frac{C}{\left(\ell\left(Q_{x, j}\right)+\ell\left(Q_{y, j+1}\right)+|x-y|\right)^{n}} .
$$

To verify (2.33), it suffices to verify that

$$
\begin{gathered}
\left|\left(E_{j} D_{k}\right)(x, y)\right| \leq \frac{C}{\ell\left(Q_{x, j}\right)^{n}}, \\
\left|\left(E_{j} D_{k}\right)(x, y)\right| \leq \frac{C}{\ell\left(Q_{y, j+1}\right)^{n}}
\end{gathered}
$$

and

$$
\left|\left(E_{j} D_{k}\right)(x, y)\right| \leq \frac{C}{|x-y|^{n}} .
$$

The size condition of $E_{j}$ (Lemma 2.4(d)) and the integral condition of $D_{k}$ (Lemma 2.4(b)) immediately yield that

$$
\begin{aligned}
\left|\left(E_{j} D_{k}\right)(x, y)\right| & =\left|\int_{\mathbb{R}^{d}} E_{j}(x, z) D_{k}(z, y) d \mu(y)\right| \\
& \leq C \int_{\mathbb{R}^{d}} \frac{1}{\left(\ell\left(Q_{x, j}\right)+\ell\left(Q_{y, j}\right)+|x-z|\right)^{n}}\left|D_{k}(z, y)\right| d \mu(z) \\
& \leq \frac{C}{\ell\left(Q_{x, j}\right)^{n}},
\end{aligned}
$$

which is (2.34).

Since $k \leq j+1$, by Lemma $2.2, Q_{y, j+1} \subset Q_{y, k}$ and, thus, $\ell\left(Q_{y, k}\right) \geq \ell\left(Q_{y, j+1}\right)$. This fact together with Lemma 2.4 tell us that

$$
\begin{aligned}
\left|\left(E_{j} D_{k}\right)(x, y)\right| & =\left|\int_{\mathbb{R}^{d}} E_{j}(x, z) D_{k}(z, y) d \mu(y)\right| \\
& \leq C \int_{\mathbb{R}^{d}}\left|E_{j}(x, z)\right| \frac{1}{\left(\ell\left(Q_{z, k}\right)+\ell\left(Q_{y, k}\right)+|z-y|\right)^{n}} d \mu(z) \\
& \leq \frac{C}{\ell\left(Q_{y, j+1}\right)^{n}},
\end{aligned}
$$

which is (2.35).

On the other hand, by Lemma 2.4, we can also obtain

$$
\begin{aligned}
& \left|\left(E_{j} D_{k}\right)(x, y)\right| \\
= & \left|\int_{\mathbb{R}^{d}} E_{j}(x, z) D_{k}(z, y) d \mu(y)\right| \\
\leq & C \int_{|x-z| \geq \frac{|x-y|}{2}} \frac{1}{\left(\ell\left(Q_{x, j}\right)+\ell\left(Q_{y, j}\right)+|x-z|\right)^{n}}\left|D_{k}(z, y)\right| d \mu(z) \\
& +C \int_{|x-z|<\frac{|x-y|}{2}}\left|E_{j}(x, z)\right| \frac{1}{\left(\ell\left(Q_{z, k}\right)+\ell\left(Q_{y, k}\right)+|z-y|\right)^{n}} d \mu(z) \\
\leq & \frac{C}{|x-y|^{n}}\left[\int_{\mathbb{R}^{d}}^{\left.\left|D_{k}(z, y)\right| d \mu(z)+\int_{\mathbb{R}^{d}}\left|E_{j}(x, z)\right| d \mu(z)\right]}\right. \\
\leq & \frac{C}{|x-y|^{n}},
\end{aligned}
$$

which is (2.36). Thus, (2.33) is true. 
It is also easy to see that $\operatorname{supp}\left(E_{j} D_{k}\right)(x, \cdot) \subset Q_{x, j-3}$ and $\operatorname{supp}\left(E_{j} D_{k}\right)(\cdot, y) \subset$ $Q_{y, j-3}$ by Lemma 2.3 and Lemma 2.4 .

Case 3. $j=k+1$. In this case, by Lemma 2.3 and Lemma 2.4 we have

$$
\operatorname{supp}\left(E_{j} D_{k}\right)(x, \cdot) \subset Q_{x, k-3}
$$

and $\operatorname{supp}\left(E_{j} D_{k}\right)(\cdot, y) \subset Q_{y, k-3}$. An argument similar to that of (2.35) tells us that

$$
\left|\left(E_{j} D_{k}\right)(x, y)\right| \leq \frac{C}{\left(\ell\left(Q_{x, k+1}\right)+\ell\left(Q_{y, k}\right)+|x-y|\right)^{n}} .
$$

Case 4. $j \leq k-2$. In this case, by Lemma 2.3 and Lemma 2.4 we have

$$
\operatorname{supp}\left(E_{j} D_{k}\right)(x, \cdot) \subset Q_{x, j-3}
$$

and $\operatorname{supp}\left(E_{j} D_{k}\right)(\cdot, y) \subset Q_{y, j-3}$. By an argument similar to that of (34) in [27, we can show that

$$
\left|\left(E_{j} D_{k}\right)(x, y)\right| \leq C 2^{-2|j-k| \theta} \frac{1}{\left(\ell\left(Q_{x, j}\right)+\ell\left(Q_{y, j}\right)+|x-y|\right)^{n}} .
$$

This finishes the proof of Lemma 2.5

Before we return to the proof of Theorem 2.1, by a result of Tolsa in 27, if $N$ is big enough, then for all $f \in L^{2}(\mu)$, we have that

$$
f=\sum_{k \in \mathbb{Z}} T_{N}^{-1} D_{k}^{N} D_{k}(f)=\sum_{k \in \mathbb{Z}} D_{k}^{N} D_{k} T_{N}^{-1}(f)
$$

holds in the norm of $L^{2}(\mu)$. In fact, $T_{N}^{-1}$ is bounded on $L^{p}(\mu)$ with $1<p<\infty$. The formula (2.37) is called the Calderón-type reproducing formula; see [27] for more details.

We now write $T_{N}^{-1}$ as

$$
T_{N}^{-1}=\sum_{l=0}^{\infty}\left(R_{N}\right)^{l}
$$

in the operator norm of $L^{2}(\mu)$, and for $l \in \mathbb{N}$,

$$
\left(R_{N}\right)^{l}=\sum_{\left|k_{1}-j_{1}\right|>N} D_{k_{1}} D_{j_{1}} \sum_{\left|k_{2}-j_{2}\right|>N} D_{k_{2}} D_{j_{2}} \ldots \sum_{\left|k_{l}-j_{l}\right|>N} D_{k_{l}} D_{j_{l}}
$$

also in the operator norm of $L^{2}(\mu)$.

We are now ready to prove Theorem 2.1.

Proof of Theorem 2.1. We only need to show (2.30). The inequality (2.32) can be deduced from (2.30) by using (2.38) and (2.39).

To verify (2.30), the formulae (2.37), (2.38) and (2.39) with $D_{k}$ replaced by $G_{k}$ tell us that for all $j \in \mathbb{Z}$,

$$
\begin{aligned}
E_{j} R_{N} f(x)= & \sum_{l=0}^{\infty} \sum_{k=-\infty}^{\infty} \sum_{i=-\infty}^{\infty} \sum_{|m|>N} \sum_{k_{1}=-\infty}^{\infty} \sum_{\left|m_{1}\right|>N_{1}} \cdots \sum_{k_{l}=-\infty}^{\infty} \\
& \times \sum_{\left|m_{l}\right|>N_{1}} E_{j} D_{i} D_{i+m} G_{k_{1}} G_{k_{1}+m_{1}} \cdots G_{k_{l}} G_{k_{l}+m_{l}} G_{k}^{N_{1}} G_{k} f(x) .
\end{aligned}
$$


By a technique used in the proof of the Cotlar-Stein lemma (see [22]) and Lemma 2.5, we obtain that for $p \in[1, \infty]$, there is a constant $C_{2}>0$ such that for all $f \in L^{2}(\mu)$

$$
\begin{aligned}
& \left\|E_{j} D_{i} D_{i+m} G_{k_{1}} G_{k_{1}+m_{1}} \cdots G_{k_{l}} G_{k_{l}+m_{l}} G_{k}^{N_{1}} G_{k} f\right\|_{L^{p}(\mu)} \\
& \leq\left\|E_{j} D_{i}\right\|_{L^{p}(\mu) \rightarrow L^{p}(\mu)}\left\|D_{i+m} G_{k_{1}}\right\|_{L^{p}(\mu) \rightarrow L^{p}(\mu)} \cdots \\
& \quad \times\left\|G_{k_{l}+m_{l}} G_{k}^{N_{1}}\right\|_{L^{p}(\mu) \rightarrow L^{p}(\mu)}\left\|G_{k} f\right\|_{L^{p}(\mu) \rightarrow L^{p}(\mu)} \\
& \leq C N_{1} C_{2}^{l} 2^{-2 \theta\left[|j-i|+\left|i+m-k_{1}\right|+\cdots+\left|k_{l}+m_{l}-k\right|\right]}\left\|G_{k} f\right\|_{L^{p}(\mu)}
\end{aligned}
$$

and

$$
\begin{aligned}
& \left\|E_{j} D_{i} D_{i+m} G_{k_{1}} G_{k_{1}+m_{1}} \cdots G_{k_{l}} G_{k_{l}+m_{l}} G_{k}^{N_{1}} G_{k} f\right\|_{L^{p}(\mu)} \\
& \leq C\left\|E_{j}\right\|_{L^{p}(\mu) \rightarrow L^{p}(\mu)}\left\|D_{i} D_{i+m}\right\|_{L^{p}(\mu) \rightarrow L^{p}(\mu)} \cdots \\
& \quad \times\left\|G_{k_{l}} G_{k_{l}+m_{l}}\right\|_{L^{p}(\mu) \rightarrow L^{p}(\mu)}\left\|G_{k}^{N_{1}}\right\|_{L^{p}(\mu) \rightarrow L^{p}(\mu)}\left\|G_{k} f\right\|_{L^{p}(\mu)} \\
& \leq C N_{1} C_{2}^{l} 2^{-2 \theta\left[|m|+\left|m_{1}\right|+\cdots+\left|m_{l}\right|\right]}\left\|G_{k} f\right\|_{L^{p}(\mu)} .
\end{aligned}
$$

Here we also used the fact that $\left\|E_{j}\right\|_{L^{p}(\mu) \rightarrow L^{p}(\mu)} \leq C$ uniformly on $j$ and

$$
\left\|G_{k}^{N_{1}}\right\|_{L^{p}(\mu) \rightarrow L^{p}(\mu)} \leq C N_{1}
$$

uniformly on $k$ with $C$ independent of $N_{1}$. The geometric means of (2.41) and (2.42) yields that

$$
\begin{aligned}
& \left\|E_{j} D_{i} D_{i+m} G_{k_{1}} G_{k_{1}+m_{1}} \cdots G_{k_{l}} G_{k_{l}+m_{l}} G_{k}^{N_{1}} G_{k} f\right\|_{L^{p}(\mu)} \\
& \leq C N_{1} C_{2}^{l} 2^{-2 \theta(1-\nu)\left[|j-i|+\left|i+m-k_{1}\right|+\cdots+\left|k_{l}+m_{l}-k\right|\right]} \\
& \quad \times 2^{-2 \theta \nu\left[|m|+\left|m_{1}\right|+\cdots+\left|m_{l}\right|\right]}\left\|G_{k} f\right\|_{L^{p}(\mu)} .
\end{aligned}
$$

From (2.40) and (2.43), it follows that

$$
\begin{aligned}
& \left\{\sum_{j=-\infty}^{\infty} 2^{j s q}\left\|E_{j} R_{N} f\right\|_{L^{p}(\mu)}^{q}\right\}^{1 / q} \\
& \leq\left\{\sum_{j=-\infty}^{\infty} 2^{j s q}\left[\sum_{l=0}^{\infty} \sum_{k=-\infty}^{\infty} \sum_{i=-\infty}^{\infty} \sum_{|m|>N} \sum_{k_{1}=-\infty}^{\infty} \sum_{\left|m_{1}\right|>N_{1}} \cdots \sum_{k_{l}=-\infty}^{\infty} \sum_{\left|m_{l}\right|>N_{1}}\left\|E_{j} D_{i} D_{i+m} G_{k_{1}} G_{k_{1}+m_{1}} \cdots G_{k_{l}} G_{k_{l}+m_{l}} G_{k}^{N_{1}} G_{k} f\right\|_{L^{p}(\mu)}\right]^{q}\right\}^{1 / q} \\
& \quad \times \sum_{l=0}^{\infty} C_{2}^{l}\left\{\sum _ { j = - \infty } ^ { \infty } \left[\sum_{i=-\infty}^{\infty} 2^{(j-i) s-2 \theta(1-\nu)|j-i|} \sum_{|m|>N} \sum_{k_{1}=-\infty}^{\infty} \sum_{k_{l} \mid>N_{1}=-\infty}^{\infty}\right.\right. \\
& \quad \times \sum_{\left|m_{l}\right|>N_{1}}^{\infty} \sum_{k=-\infty}^{\infty} 2^{i s} 2^{-2 \theta(1-\nu)\left[\left|i+m-k_{1}\right|+\cdots+\left|k_{l}+m_{l}-k\right|\right]} \\
& \left.\quad \times 2^{-2 \theta \nu\left[|m|+\left|m_{1}\right|+\cdots+\left|m_{l}\right|\right]}\left\|G_{k} f\right\|_{L^{p}(\mu)}\right]^{q / q}
\end{aligned}
$$


Using the fact that $|s|<2(1-\nu) \theta$ and the Hölder inequality on the summation of $i$, and then exchanging the order of the summation on $i$ with the summation on $j$, we then know that the last quantity in the above inequality can be controlled by

$$
\begin{aligned}
& \leq C \sum_{l=0}^{\infty} C_{2}^{l}\left\{\sum _ { j = - \infty } ^ { \infty } \left(\left[\sum _ { i = - \infty } ^ { \infty } 2 ^ { ( j - i ) s - 2 \theta ( 1 - \nu ) | j - i | } \left(\sum_{|m|>N} \sum_{k_{1}=-\infty}^{\infty} \sum_{\left|m_{1}\right|>N_{1}} \ldots \sum_{k_{l}=-\infty}^{\infty}\right.\right.\right.\right. \\
& \times \sum_{\left|m_{l}\right|>N_{1}} \sum_{k=-\infty}^{\infty} 2^{i s} 2^{-2 \theta(1-\nu)\left[\left|i+m-k_{1}\right|+\cdots+\left|k_{l}+m_{l}-k\right|\right]}
\end{aligned}
$$

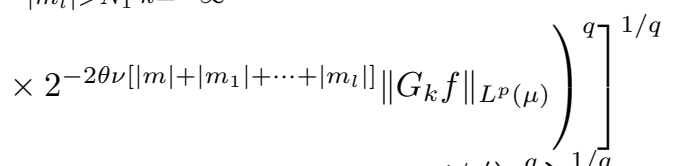

$$
\begin{aligned}
& \left.\left.\times\left[\sum_{i=-\infty}^{\infty} 2^{(j-i) s-2 \theta(1-\nu)|j-i|}\right]^{1 / q^{\prime}}\right)^{q}\right\}^{1 / q} \\
& \leq C \sum_{l=0}^{\infty} C_{2}^{l}\left\{\sum _ { i = - \infty } ^ { \infty } ( \sum _ { j = - \infty } ^ { \infty } 2 ^ { ( j - i ) s - 2 \theta ( 1 - \nu ) | j - i | } ) \left[\sum_{|m|>N} \sum_{k_{1}=-\infty}^{\infty} \sum_{\left|m_{1}\right|>N_{1}} \ldots \sum_{k_{l}=-\infty}^{\infty}\right.\right. \\
& \times \sum_{\left|m_{l-1}\right|>N_{1}} \sum_{k=-\infty}^{\infty} \sum_{\left|m_{l}\right|>N_{1}} 2^{i s} 2^{-2 \theta(1-\nu)\left[\left|i+m-k_{1}\right|+\cdots+\left|k_{l}+m_{l}-k\right|\right]} \\
& \left.\left.\times 2^{-2 \theta \nu\left[|m|+\left|m_{1}\right|+\cdots+\left|m_{l}\right|\right]}\left\|G_{k} f\right\|_{L^{p}(\mu)}\right]^{q}\right\}^{1 / q} \\
& \leq C \sum_{l=0}^{\infty} C_{2}^{l}\left\{\sum _ { i = - \infty } ^ { \infty } \left[\sum_{k_{1}=-\infty}^{\infty} 2^{\left(i+m-k_{1}\right) s-2 \theta(1-\nu)\left|i+m-k_{1}\right|} \sum_{|m|>N} \sum_{k_{2}=-\infty}^{\infty} \sum_{\left|m_{1}\right|>N_{1}} \ldots\right.\right. \\
& \times \sum_{k_{l}=-\infty}^{\infty} \sum_{\left|m_{l-1}\right|>N_{1}} \sum_{k=-\infty}^{\infty} \sum_{\left|m_{l}\right|>N_{1}} 2^{\left(k_{1}-m\right) s} 2^{-2 \theta(1-\nu)\left[\left|k_{1}+m_{1}-k_{2}\right|+\cdots+\left|k_{l}+m_{l}-k\right|\right]} \\
& \left.\left.\times 2^{-2 \theta \nu\left[|m|+\left|m_{1}\right|+\cdots+\left|m_{l}\right|\right]}\left\|G_{k} f\right\|_{L^{p}(\mu)}\right]^{q}\right\}^{1 / q}
\end{aligned}
$$

For the summation on $k_{1}$ and the summation on $i$, repeating the same procedure as that on the summation on $i$ and the summation on $j$, that is, using the fact that $|s|<2(1-\nu) \theta$ and the Hölder inequality on the summation of $k_{1}$ and then exchanging the order of the summation on $k_{1}$ with the summation on $i$, we find that the last term in the above inequality is dominated by

$$
\begin{aligned}
\leq & C \sum_{l=0}^{\infty} C_{2}^{l}\left\{\sum _ { k _ { 1 } = - \infty } ^ { \infty } \left[\sum_{|m|>N} 2^{-m s-2 \theta \nu|m|} \sum_{k_{2}=-\infty}^{\infty} \sum_{\left|m_{1}\right|>N_{1}} \ldots\right.\right. \\
& \times \sum_{k_{l}=-\infty}^{\infty} \sum_{\left|m_{l-1}\right|>N_{1}} \sum_{k=-\infty}^{\infty} \sum_{\left|m_{l}\right|>N_{1}} 2^{k_{1} s} 2^{-2 \theta(1-\nu)\left[\left|k_{1}+m_{1}-k_{2}\right|+\cdots+\left|k_{l}+m_{l}-k\right|\right]} \\
& \left.\left.\times 2^{-2 \theta \nu\left[\left|m_{1}\right|+\cdots+\left|m_{l}\right|\right]}\left\|G_{k} f\right\|_{L^{p}(\mu)}\right]^{q}\right\}^{1 / q} .
\end{aligned}
$$


The fact $|s|<2 \theta \nu$ and the summation on $m$ tell us that the above quantity can be controlled by

$$
\begin{aligned}
\leq & \widetilde{C}_{2}\left(2^{-N(s+2 \theta \nu)}+2^{-N(2 \theta \nu-s)}\right) \sum_{l=0}^{\infty} C_{2}^{l}\left\{\sum _ { k _ { 1 } = - \infty } ^ { \infty } \left[\sum_{k_{2}=-\infty}^{\infty} \sum_{\left|m_{1}\right|>N_{1}} \ldots\right.\right. \\
& \times \sum_{k_{l}=-\infty}^{\infty} \sum_{\left|m_{l-1}\right|>N_{1}} \sum_{k=-\infty}^{\infty} \sum_{\left|m_{l}\right|>N_{1}} 2^{k_{1} s} 2^{-2 \theta(1-\nu)\left[\left|k_{1}+m_{1}-k_{2}\right|+\cdots+\left|k_{l}+m_{l}-k\right|\right]} \\
& \times 2^{\left.\left.-2 \theta \nu\left[\left|m_{1}\right|+\cdots+\left|m_{l}\right|\right]\left\|G_{k} f\right\|_{L^{p}(\mu)}\right]^{q}\right\}^{1 / q}},
\end{aligned}
$$

where $\widetilde{C}_{2}$ is a positive constant independent of $N$. Repeating this procedure $l$ times, we finally obtain that the above term is dominated by

$$
\begin{aligned}
\leq & \widetilde{C}_{2}\left(2^{-N(s+2 \theta \nu)}+2^{-N(2 \theta \nu-s)}\right) \\
& \times\left\{\sum_{l=0}^{\infty}\left(\widetilde{C}_{2} C_{2}\right)^{l}\left(2^{-N_{1}(s+2 \theta \nu)}+2^{-N_{1}(2 \theta \nu-s)}\right)^{l}\right\}\left\{\sum_{k=-\infty}^{\infty} 2^{k s q}\left\|G_{k} f\right\|_{L^{p}(\mu)}^{q}\right\}^{1 / q} .
\end{aligned}
$$

If we now choose $N_{1} \in \mathbb{N}$ large enough so that

$$
\widetilde{C}_{2}\left(2^{-N_{1}(s+2 \theta \nu)}+2^{-N_{1}(2 \theta \nu-s)}\right)<1
$$

and then let

$$
C_{1}=\widetilde{C}_{2}\left\{\sum_{l=0}^{\infty}\left(\widetilde{C}_{2} C_{2}\right)^{l}\left(2^{-N_{1}(s+2 \theta \nu)}+2^{-N_{1}(2 \theta \nu-s)}\right)^{l}\right\},
$$

we obtain (2.30).

This finishes the proof of Theorem 2.1 .

We now use the approximation to the identity in Definition 2.9 to introduce the space of test functions.

Definition 2.10. Let $\left\{S_{k}\right\}_{k \in \mathbb{Z}}$ be an approximation to the identity as in Definition 2.9. $D_{k}=S_{k}-S_{k-1}$ for $k \in \mathbb{Z}$ and let $\theta$ be the same as in Definition 2.5. For $|s|<\theta$, $1 \leq p, q \leq \infty$ and $f \in L^{2}(\mu)$, we define

$$
\|f\|_{\dot{B}_{p q}^{s}(\mu)}=\left\{\sum_{k=-\infty}^{\infty} 2^{k s q}\left\|D_{k} f\right\|_{L^{p}(\mu)}^{q}\right\}^{1 / q}
$$

and

$$
\dot{\mathcal{B}}_{p q}^{s}(\mu)=\left\{f \in L^{2}(\mu):\|f\|_{\dot{B}_{p q}^{s}(\mu)}<\infty\right\} .
$$

Applying Theorem 2.1, we can show that the space of test functions, $\dot{\mathcal{B}}_{p q}^{s}(\mu)$, in Definition 2.10 is independent of the chosen approximations to the identity.

Proposition 2.1. Let $\theta$ be the same as in Definition 2.5. Let $\left\{S_{k}\right\}_{k \in \mathbb{Z}}$ and $\left\{P_{k}\right\}_{k \in \mathbb{Z}}$ be two approximations to the identity as in Definition [2.9. Set $D_{k}=S_{k}-S_{k-1}$ and 
$E_{k}=P_{k}-P_{k-1}$ for $k \in \mathbb{Z}$. Then for $|s|<\theta$ and $1 \leq p, q \leq \infty$, and all $f \in L^{2}(\mu)$,

$$
\left\{\sum_{k=-\infty}^{\infty} 2^{k s q}\left\|D_{k} f\right\|_{L^{p}(\mu)}^{q}\right\}^{1 / q} \sim\left\{\sum_{k=-\infty}^{\infty} 2^{k s q}\left\|E_{k} f\right\|_{L^{p}(\mu)}^{q}\right\}^{1 / q} .
$$

Proof. For given $|s|<\theta$, we choose $\nu \in(0,1 / 2)$ such that $|s|<2 \nu \theta$. By (2.37), for any $j \in \mathbb{Z}$, we can write

$$
E_{j} f(x)=\sum_{k=-\infty}^{\infty} E_{j} D_{k}^{N} D_{k} T_{N}^{-1}(f)(x),
$$

where $N \in \mathbb{N}$ is large enough such that (2.31) holds.

Then the Minkowski inequality, Lemma 2.5 and Theorem 2.1 yield

$$
\begin{aligned}
& \left\{\sum_{j=-\infty}^{\infty} 2^{j s q}\left\|E_{j} f\right\|_{L^{p}(\mu)}^{q}\right\}^{1 / q} \\
& \leq\left\{\sum_{j=-\infty}^{\infty} 2^{j s q}\left[\sum_{k=-\infty}^{\infty}\left\|E_{j} D_{k}^{N} D_{k} T_{N}^{-1}(f)\right\|_{L^{p}(\mu)}\right]^{q}\right\}^{1 / q} \\
& \leq C\left\{\sum_{j=-\infty}^{\infty}\left[\sum_{k=-\infty}^{\infty} 2^{(j-k) s-2 \theta|j-k|} 2^{k s}\left\|D_{k} T_{N}^{-1}(f)\right\|_{L^{p}(\mu)}\right]^{q}\right\}^{1 / q} \\
& \leq C\left\{\sum _ { j = - \infty } ^ { \infty } \left[\left(\sum_{k=-\infty}^{\infty} 2^{(j-k) s-2 \theta|j-k|} 2^{k s q}\left\|D_{k} T_{N}^{-1}(f)\right\|_{L^{p}(\mu)}^{q}\right)^{1 / q}\right.\right. \\
& \left.\left.\times\left(\sum_{k=-\infty}^{\infty} 2^{(j-k) s-2 \theta|j-k|}\right)^{1 / q^{\prime}}\right]^{q}\right\}^{1 / q} \\
& \leq C\left\{\sum_{k=-\infty}^{\infty}\left(\sum_{j=-\infty}^{\infty} 2^{(j-k) s-2 \theta|j-k|}\right)^{\left.2^{k s q}\left\|D_{k} T_{N}^{-1}(f)\right\|_{L^{p}(\mu)}^{q}\right\}^{1 / q}}\right. \\
& =C\left\{\sum_{k=-\infty}^{\infty} 2^{k s q}\left\|D_{k} T_{N}^{-1}(f)\right\|_{L^{p}(\mu)}^{q}\right\}^{1 / q} \\
& \leq C\left\{\sum_{k=-\infty}^{\infty} 2^{k s q}\left\|D_{k} f\right\|_{L^{p}(\mu)}^{q}\right\}^{1 / q} \cdot
\end{aligned}
$$

By symmetry, we have then finished the proof of Proposition 2.1 .

The following theorem is one of the main results of this paper.

Theorem 2.2. Let $\theta$ be the same as in Definition 2.5, and let $\left\{D_{k}\right\}_{k \in \mathbb{Z}}$ be the same as in Theorem 2.1. If $1 \leq p \leq \infty$ and $1 \leq q<\infty$, then for all $f \in \dot{\mathcal{B}}_{p q}^{s}(\mu)$,

$$
f=\sum_{k \in \mathbb{Z}} D_{k}^{N} D_{k} T_{N}^{-1}(f)=\sum_{k \in \mathbb{Z}} T_{N}^{-1} D_{k}^{N} D_{k}(f)
$$


holds in both the norm $\|\cdot\|_{\dot{B}_{p q}^{s}(\mu)}$ and the norm $\|\cdot\|_{\dot{B}_{p \infty}^{s}(\mu)} \cdot$ Moreover, for all $g \in \dot{\mathcal{B}}_{p q}^{s}(\mu)$ with $1 \leq p, q<\infty$,

$$
\langle f, g\rangle=\sum_{k \in \mathbb{Z}}\left\langle D_{k} D_{k}^{N} T_{N}^{-1}(f), g\right\rangle=\sum_{k \in \mathbb{Z}}\left\langle T_{N}^{-1} D_{k} D_{k}^{N}(f), g\right\rangle
$$

holds for all $f \in\left(\dot{\mathcal{B}}_{p q}^{s}(\mu)\right)^{\prime}$ with $1 \leq p, q \leq \infty$.

Proof. We only show the first equality in (2.45). The proof for the second equality in (2.45) is similar. The proof that (2.45) holds in the norm $\|\cdot\|_{\dot{B}_{p \infty}^{s}(\mu)}$ is easy by noting that $\dot{\mathcal{B}}_{p q}^{s}(\mu) \subset \dot{\mathcal{B}}_{p \infty}^{s}(\mu)$ for $1 \leq q<\infty$, which is a simple result of the monotonicity of $l^{q}$; see the proof of Proposition 2.3.2/2 in [31, p. 47].

Let $f \in \dot{\mathcal{B}}_{p q}^{s}(\mu), 1 \leq p \leq \infty$ and $1 \leq q<\infty$. Then, it suffices to show that

$$
\lim _{L \rightarrow \infty}\left\|\sum_{|k|>L} D_{k}^{N} D_{k} T_{N}^{-1}(f)\right\|_{\dot{B}_{p q}^{s}(\mu)}=0 .
$$

Lemma 2.5, the Minkowski inequality, the Hölder inequality and Theorem 2.1 lead to

$$
\begin{aligned}
& \left\|\sum_{|k|>L} D_{k}^{N} D_{k} T_{N}^{-1}(f)\right\|_{\dot{B}_{p q}^{s}(\mu)} \\
= & \left\{\sum_{j=-\infty}^{\infty} 2^{j s q}\left\|D_{j}\left(\sum_{|k|>L} D_{k}^{N} D_{k} T_{N}^{-1}(f)\right)\right\|_{L^{p}(\mu)}^{q}\right\}^{1 / q} \\
\leq & C\left\{\sum_{j=-\infty}^{\infty}\left[\sum_{|k|>L} 2^{(j-k) s-2|j-k| \theta} 2^{k s}\left\|D_{k} T_{N}^{-1}(f)\right\|_{L^{p}(\mu)}\right]^{q}\right\}^{1 / q} \\
\leq & C\left\{\sum _ { j = - \infty } ^ { \infty } \left[\left(\sum_{|k|>L} 2^{(j-k) s-2|j-k| \theta} 2^{k s q}\left\|D_{k} T_{N}^{-1}(f)\right\|_{L^{p}(\mu)}^{q}\right)^{1 / q}\right.\right. \\
& \left.\left.\times\left(\sum_{|k|>L} 2^{(j-k) s-2|j-k| \theta}\right)^{1 / q^{\prime}}\right]^{q}\right\}^{1 / q} \\
\leq & C\left\{\sum_{|k|>L}\left[\sum_{j=-\infty}^{\infty} 2^{(j-k) s-2|j-k| \theta}\right]^{k s q}\left\|D_{k} T_{N}^{-1}(f)\right\|_{L^{p}(\mu)}^{q}\right\}^{1 / q} \\
\leq & C\left\{\sum_{|k|>L} 2^{k s q}\left\|D_{k} T_{N}^{-1}(f)\right\|_{L^{p}(\mu)}^{q}\right\}^{1 / q} \\
\rightarrow & 0
\end{aligned}
$$

as $L \rightarrow \infty$, since $T_{N}^{-1}(f) \in \dot{\mathcal{B}}_{p q}^{s}(\mu)$. Thus, (2.47) holds and therefore, the first equality in (2.45) holds. 
From (2.45) we can deduce the second equality in (2.46). In fact, for all $g \in$ $\dot{\mathcal{B}}_{p q}^{s}(\mu)$ with $1 \leq p, q<\infty$, then

$$
\langle f, g\rangle=\left\langle f, \sum_{k \in \mathbb{Z}} D_{k}^{N} D_{k} T_{N}^{-1}(g)\right\rangle=\sum_{k \in \mathbb{Z}}\left\langle f, D_{k}^{N} D_{k} T_{N}^{-1}(g)\right\rangle,
$$

where $f \in\left(\dot{\mathcal{B}}_{p q}^{s}(\mu)\right)^{\prime}$ with $1 \leq p, q \leq \infty$.

To finish the proof, we only need to verify that for any $k \in \mathbb{Z}$,

$$
\left\langle f, D_{k}^{N} D_{k} T_{N}^{-1}(g)\right\rangle=\left\langle D_{k} D_{k}^{N} T_{N}^{-1}(f), g\right\rangle .
$$

To this end, for any $M>0$, let $Q_{0, M}$ be the cube centered at the origin with the side length $2 M$. Define

$$
g_{k, M}(x)=\int_{Q_{0, M}} D_{k}^{N}(x, y)\left(D_{k} T_{N}^{-1}\right)(g)(y) d \mu(y) .
$$

We claim that

$$
\lim _{M \rightarrow \infty}\left\|D_{k}^{N} D_{k} T_{N}^{-1}(g)-g_{k, M}\right\|_{\dot{B}_{p q}^{s}(\mu)}=0 .
$$

In fact, Theorem 2.1 tells us that $T_{N}^{-1} g \in \dot{B}_{p q}^{s}(\mu)$ and Lemma 2.5 yields

$$
\begin{aligned}
& \left\|D_{k}^{N} D_{k} T_{N}^{-1}(g)-g_{k, M}\right\|_{\dot{B}_{p q}^{s}(\mu)} \\
& =\left\{\sum_{l=-\infty}^{\infty} 2^{l s q}\left\|D_{l}\left[\int_{\mathbb{R}^{d} \backslash Q_{0, M}} D_{k}^{N}(\cdot, y)\left(D_{k} T_{N}^{-1}\right)(g)(y) d \mu(y)\right]\right\|_{L^{p}(\mu)}^{q}\right\}^{1 / q} \\
& \leq\left\{\sum_{l=-\infty}^{\infty} 2^{l s q}\left\|D_{l} D_{k}^{N}\right\|_{L^{p}(\mu) \rightarrow L^{p}(\mu)}^{q}\left[\int_{\mathbb{R}^{d} \backslash Q_{0, M}}\left|\left(D_{k} T_{N}^{-1}\right)(g)(y)\right|^{p} d \mu(y)\right]^{q / p}\right\}^{1 / q} \\
& \leq C N\left\{\sum_{l=-\infty}^{\infty} 2^{[(l-k) s-2|k-l| \theta] q}\right\}^{1 / q} 2^{k s}\left[\int_{\mathbb{R}^{d} \backslash Q_{0, M}}\left|\left(D_{k} T_{N}^{-1}\right)(g)(y)\right|^{p} d \mu(y)\right]^{1 / p} \\
& \leq C N 2^{k s}\left[\int_{\mathbb{R}^{d} \backslash Q_{0, M}}\left|\left(D_{k} T_{N}^{-1}\right)(g)(y)\right|^{p} d \mu(y)\right]^{1 / p} \\
& \rightarrow 0,
\end{aligned}
$$

as $M \rightarrow \infty$. Thus, (2.49) holds. Therefore,

$$
\left\langle f, D_{k}^{N} D_{k} T_{N}^{-1}(g)\right\rangle=\lim _{M \rightarrow \infty}\left\langle f, g_{k, M}\right\rangle .
$$

Let $S=Q_{0, M} \cap \operatorname{supp}(\mu)$. For any $z \in S$, there is a cube $Q_{z, k+N}$ centered at $z$. Thus, $\left\{Q_{z, k+N}\right\}_{z \in S}$ is a covering of $S$. By the compactness of $S$, we can find a finite number of cubes, $\left\{Q_{z_{i}, k+N}\right\}_{i=1}^{\nu} \subset\left\{Q_{z, k+N}\right\}_{z \in S}$, such that $\bigcup_{i=1}^{\nu} Q_{z_{i}, k+N} \supset S$. We now decompose $S$ into the union of a finite number of cubes with disjoint interior, $\left\{Q_{j}\right\}_{j=1}^{N_{0}}$, such that each $Q_{j}$ for $j \in\left\{1, \cdots, N_{0}\right\}$ is contained in some $Q_{z_{i}, k+N}$ for some $i \in\{1, \cdots, \nu\}$. We then divide each $Q_{j}$ into a union of cubes, $\left\{Q_{j}^{i}\right\}_{i=1}^{N_{j}}$, such 
that $\ell\left(Q_{j}^{i}\right) \sim 2^{-J}$, where $N_{j} \sim 2^{J} \ell\left(Q_{j}\right)$ for $j=1, \cdots, N_{0}$. Now we write

$$
\begin{aligned}
g_{k, M}(x)= & \sum_{j=1}^{N_{0}} \int_{Q_{j}} D_{k}^{N}(x, y)\left(D_{k} T_{N}^{-1}\right)(g)(y) d \mu(y) \\
= & \sum_{j=1}^{N_{0}} \sum_{i=1}^{N_{j}} \int_{Q_{j}^{i}}\left[D_{k}^{N}(x, y)-D_{k}^{N}\left(x, y_{Q_{j}^{i}}\right)\right]\left(D_{k} T_{N}^{-1}\right)(g)(y) d \mu(y) \\
& +\sum_{j=1}^{N_{0}} \sum_{i=1}^{N_{j}} D_{k}^{N}\left(x, y_{Q_{j}^{i}}\right) \int_{Q_{j}^{i}}\left(D_{k} T_{N}^{-1}\right)(g)(y) d \mu(y) \\
= & g_{k, M}^{1}(x)+g_{k, M}^{2}(x),
\end{aligned}
$$

where $y_{Q_{j}^{i}}$ is any point in the cube $Q_{j}^{i}$. We now claim that for any fixed $k$ and $M$,

$$
\lim _{J \rightarrow \infty}\left\|g_{k, M}^{1}\right\|_{\dot{B}_{p q}^{s}(\mu)}=0 .
$$

To prove this claim, the second difference smoothness condition will be used. Let

$$
F_{k, i, j}(z, y)=\left[D_{k}^{N}(z, y)-D_{k}^{N}\left(z, y_{Q_{j}^{i}}\right)\right] \chi_{Q_{j}^{i}}(y) .
$$

Lemmas 2.4 and 2.5 tell us that

$$
\begin{gathered}
\operatorname{supp} F_{k, i, j}(\cdot, y) \subset Q_{y, k-N-3}, \quad \operatorname{supp} F_{k, i, j}(z, \cdot) \subset Q_{z, k-N-3} ; \\
\int_{\mathbb{R}^{d}} F_{k, i, j}(z, y) d \mu(z)=0 ; \\
\left|F_{k, i, j}(z, y)\right| \leq C_{3} 2^{-J} \ell\left(Q_{z_{i_{0}}, k+N}\right)^{-1} \frac{1}{\left(\ell\left(Q_{z, k+N}\right)+\ell\left(Q_{y, k+N}\right)+|z-y|\right)^{n}}
\end{gathered}
$$

if $Q_{j}^{i} \subset Q_{z_{i_{0}}, k+N}$ for some $i_{0} \in\{1, \cdots, \nu\}$; and

$$
\begin{aligned}
& \left|F_{k, i, j}(z, y)-F_{k, i, j}\left(z^{\prime}, y\right)\right| \\
& \leq C_{3} 2^{-J} \ell\left(Q_{z_{0}, k+N}\right)^{-1} \frac{\left|z-z^{\prime}\right|}{\ell\left(Q_{x_{0}, k+N}\right)} \frac{1}{\left(\ell\left(Q_{z, k+N}\right)+\ell\left(Q_{y, k+N}\right)+|z-y|\right)^{n}}
\end{aligned}
$$

if $z, z^{\prime} \in Q_{x_{0}, k+N}$ for some $x_{0} \in \operatorname{supp}(\mu)$ and $Q_{j}^{i} \subset Q_{z_{i_{0}}, k+N}$ for some $i_{0} \in$ $\{1, \cdots, \nu\}$. Here $C_{3}$ depends on $N$. From (2.52), (2.53), (2.54), (2.55), Lemma 2.5 and its proof, it follows that for all $l, k \in \mathbb{Z}$ and $x, y \in \operatorname{supp}(\mu)$,

$$
\begin{aligned}
& \operatorname{supp}\left(D_{l} F_{k, i, j}\right)(\cdot, y) \subset Q_{y, \min (l, k-N-1)-3}, \\
& \operatorname{supp}\left(D_{l} F_{k, i, j}\right)(x, \cdot) \subset Q_{x, \min (l, k-N-1)-3},
\end{aligned}
$$

and for all $x \in \operatorname{supp}(\mu)$ and $y \in Q_{j}^{i} \subset Q_{z_{i_{0}}, k+N}$ for some $i_{0} \in\{1, \cdots, \nu\}$,

$$
\begin{aligned}
\left|\left(D_{l} F_{k, i, j}\right)(x, y)\right| \leq & C_{3} 2^{-J} 2^{-2|l-k| \theta} \ell\left(Q_{z_{0}, k+N}\right)^{-1} \\
& \times \frac{1}{\left(\ell\left(Q_{x, \min (l, k+N)+1}\right)+\ell\left(Q_{y, \min (l, k+N)+1}\right)+|x-y|\right)^{n}} .
\end{aligned}
$$

Let

$$
C_{4}=\max \left\{C_{3} \frac{1}{\ell\left(Q_{z_{i}, k+N}\right)}: i=1, \cdots, \nu\right\}
$$


Then $C_{4}$ depends on $N, k$, but not on $J$ and $l$. Set

$$
K(x, y)=\sum_{j=1}^{N_{0}} \sum_{i=1}^{N_{j}}\left(D_{l} F_{k, i, j}\right)(x, y) .
$$

Then, by (2.57) and (2.58), we have

$$
\begin{aligned}
& \int_{\mathbb{R}^{d}}|K(x, y)| d \mu(y) \leq C C_{4} 2^{-J} 2^{-2|l-k| \theta} \\
& \times \sum_{j=1}^{N_{0}} \sum_{i=1}^{N_{j}} \int_{Q_{j}^{i} \cap Q_{x, \min (l, k-N-1)-3}} \frac{1}{\left(\ell\left(Q_{x, \min (l, k+N)+1}\right)+|x-y|\right)^{n}} d \mu(y) \\
& =C C_{4} 2^{-J} 2^{-2|l-k| \theta} \\
& \times \sum_{j=1}^{N_{0}} \int_{Q_{j} \cap Q_{x, \min (l, k-N-1)-3}} \frac{1}{\left(\ell\left(Q_{x, \min (l, k+N)+1}\right)+|x-y|\right)^{n}} d \mu(y) \\
& \leq C C_{4} N_{0} 2^{-J} 2^{-2|l-k| \theta}\left\{\int_{Q_{x, \min (l, k+N)+1}} \frac{d \mu(y)}{\ell\left(Q_{x, \min (l, k+N)+1}\right)^{n}}\right. \\
& \left.+\delta\left(Q_{x, \min (l, k-N-1)-3}, Q_{x, \min (l, k+N)+1}\right)\right\} \\
& \leq C_{5} 2^{-J} 2^{-2|l-k| \theta},
\end{aligned}
$$

and, similarly, by (2.56) and (2.58),

$$
\int_{\mathbb{R}^{d}}|K(x, y)| d \mu(x) \leq C_{5} 2^{-J} 2^{-2|l-k| \theta},
$$

where $C_{5}$ is independent of $J$ and $l$, but, it may depend on $M, N$ and $k$. Therefore, from Schur's Lemma together with (2.59) and (2.60), it follows that

$$
\left\|D_{l}\left(g_{k, M}^{1}\right)\right\|_{L^{p}(\mu)} \leq C_{5} 2^{-J} 2^{-2|l-k| \theta}\left\|\left(D_{k} T_{N}^{-1}\right)(g)\right\|_{L^{p}(\mu)},
$$

and, from this, it further follows that

$$
\begin{aligned}
\left\|g_{k, M}^{1}\right\|_{\dot{B}_{p q}^{s}(\mu)} & \leq C_{5} 2^{-J}\left\{\sum_{l=-\infty}^{\infty} 2^{l s q} 2^{-2|l-k| \theta q}\right\}^{1 / q}\left\|\left(D_{k} T_{N}^{-1}\right)(g)\right\|_{L^{p}(\mu)} \\
& \leq C C_{5} 2^{-J} 2^{k s}\left\|\left(D_{k} T_{N}^{-1}\right)(g)\right\|_{L^{p}(\mu)} \\
& \rightarrow 0
\end{aligned}
$$

as $J \rightarrow \infty$. Obviously, (2.61) implies (2.51). By (2.50) and (2.51), we have

$$
\begin{aligned}
\left\langle f, D_{k}^{N} D_{k} T_{N}^{-1}(g)\right\rangle & =\lim _{M \rightarrow \infty}\left\langle f, g_{k, M}\right\rangle \\
& =\lim _{M \rightarrow \infty} \lim _{J \rightarrow \infty}\left\langle f, g_{k, M}^{2}\right\rangle \\
& =\lim _{M \rightarrow \infty} \lim _{J \rightarrow \infty} \sum_{j=1}^{N_{0}} \sum_{i=1}^{N_{j}} D_{k}^{N}(f)\left(y_{Q_{j}^{i}}\right) \int_{Q_{j}^{i}}\left(D_{k} T_{N}^{-1}\right)(g)(y) d \mu(y) .
\end{aligned}
$$


We now write

$$
\begin{aligned}
& \sum_{i=1}^{N_{j}} D_{k}^{N}(f)\left(y_{Q_{j}^{i}}\right) \int_{Q_{j}^{i}}\left(D_{k} T_{N}^{-1}\right)(g)(y) d \mu(y) \\
& \quad=\sum_{i=1}^{N_{j}} \int_{Q_{j}^{i}} D_{k}^{N}(f)(y)\left(D_{k} T_{N}^{-1}\right)(g)(y) d \mu(y) \\
& \quad+\int_{\mathbb{R}^{d}}\left\{\sum_{i=1}^{N_{j}}\left[D_{k}^{N}(f)\left(y_{Q_{j}^{i}}\right)-D_{k}^{N}(f)(y)\right] \chi_{Q_{j}^{i}}(y)\right\}\left(D_{k} T_{N}^{-1}\right)(g)(y) d \mu(y) .
\end{aligned}
$$

Using the second difference property of the approximation to the identity in Lemma 2.4(f), by a proof similar to that for $(2.61)$, we can show that

$$
\left\|\sum_{i=1}^{N_{j}}\left[D_{k}^{N}\left(y_{Q_{j}^{i}}, \cdot\right)-D_{k}^{N}(y, \cdot)\right] \chi_{Q_{j}^{i}}(\cdot)\right\|_{\dot{B}_{p q}^{s}(\mu)} \leq C_{6} 2^{-J}
$$

where $C_{6}$ is independent of $J$. From this, it follows that

$$
\left|\sum_{i=1}^{N_{j}}\left[D_{k}^{N}(f)\left(y_{Q_{j}^{i}}\right)-D_{k}^{N}(f)(y)\right] \chi_{Q_{j}^{i}}(y)\right| \leq C_{6} 2^{-J}\|f\|_{\left(\dot{\mathcal{B}}_{p q}^{s}(\mu)\right)^{\prime}}
$$

for all $y \in \operatorname{supp}(\mu)$. Noting that $\left(D_{k} T_{N}^{-1}\right)(g) \in L^{q}(\mu)$ by Theorem 2.1 and the construction of $\left\{Q_{j}^{i}\right\}$ for $j \in\left\{1, \cdots, N_{0}\right\}$ and $i \in\left\{1, \cdots, N_{j}\right\}$, by the Lebesgue dominated convergence theorem, we have

$$
\lim _{J \rightarrow \infty} \int_{\mathbb{R}^{d}}\left\{\sum_{i=1}^{N_{j}}\left[D_{k}^{N}(f)\left(y_{Q_{j}^{i}}\right)-D_{k}^{N}(f)(y)\right] \chi_{Q_{j}^{i}}(y)\right\}\left(D_{k} T_{N}^{-1}\right)(g)(y) d \mu(y)=0 .
$$

Thus, by this fact together with (2.62), we further have

$$
\begin{aligned}
\left\langle f, D_{k}^{N} D_{k} T_{N}^{-1}(g)\right\rangle & =\lim _{M \rightarrow \infty} \lim _{J \rightarrow \infty} \sum_{j=1}^{N_{0}} \sum_{i=1}^{N_{j}} \int_{Q_{j}^{i}} D_{k}^{N}(f)(y)\left(D_{k} T_{N}^{-1}\right)(g)(y) d \mu(y) \\
& =\int_{\mathbb{R}^{d}} D_{k}^{N}(f)(y)\left(D_{k} T_{N}^{-1}\right)(g)(y) d \mu(y) \\
& =\left\langle T_{N}^{-1} D_{k} D_{k}^{N}(f), g\right\rangle .
\end{aligned}
$$

That is, (2.48) holds, and we have completed the proof of Theorem 2.2.

\section{BESOV SPACES}

It is easy to see that $D_{k}(x, \cdot) \in L^{2}(\mu)$ with compact support for all $x \in \operatorname{supp}(\mu)$ and all $k \in \mathbb{Z}$. Let $|s|<\theta$. We will show that $D_{k}(x, \cdot) \in \dot{\mathcal{B}}_{p q}^{s}(\mu)$ for all $x \in \operatorname{supp}(\mu)$ and $1 \leq p, q \leq \infty$.

Lemma 3.1. Let $\theta$ be the same as in Definition 2.5, $|s|<\theta$ and $1 \leq p, q \leq \infty$. Let $\left\{D_{k}\right\}_{k=-\infty}^{\infty}$ be the same as in Theorem 2.1. Then $D_{k}(x, \cdot)$ and $D_{k}(\cdot, x)$ are in $\dot{\mathcal{B}}_{p q}^{s}(\mu)$ for all $x \in \operatorname{supp}(\mu)$ and all $k \in \mathbb{Z}$. 
Proof. Noting that $D_{k}(x, \cdot)=D_{k}(\cdot, x)$, we only need to verify the lemma for $D_{k}(x, \cdot)$. For any $k \in \mathbb{Z}$, any $x \in \operatorname{supp}(\mu)$ and $p=1$, by Lemma 2.5. we first have

$$
\begin{aligned}
\left\|D_{j} D_{k}(\cdot, x)\right\|_{L^{p}(\mu)} \leq & C 2^{-2|j-k| \theta} \int_{Q_{x, \min (j, k)-3}} \frac{1}{\left(\ell\left(Q_{x, \min (j, k)+1}\right)+|y-x|\right)^{n}} d \mu(y) \\
\leq & C 2^{-2|j-k| \theta}\left\{\int_{Q_{x, \min (j, k)+1}} \frac{1}{\left(\ell\left(Q_{x, \min (j, k)+1}\right)+|y-x|\right)^{n}} d \mu(y)\right. \\
& \left.+\int_{Q_{x, \min (j, k)-3} \backslash Q_{x, \min (j, k)+1}} \cdots\right\} \\
\leq & C 2^{-2|j-k| \theta} \delta\left(Q_{x, \min (j, k)+1}, Q_{x, \min (j, k)+1}\right) \\
\leq & C 2^{-2|j-k| \theta}
\end{aligned}
$$

if $p=\infty$, it is obvious that we have

$$
\left\|D_{j} D_{k}(\cdot, x)\right\|_{L^{p}(\mu)} \leq C 2^{-2|j-k| \theta} \frac{1}{\ell\left(Q_{x, k+1}\right)^{n}} ;
$$

and if $1<p<\infty$, then

$$
\begin{aligned}
\left\|D_{j} D_{k}(\cdot, x)\right\|_{L^{p}(\mu)} & \leq\left\|D_{j} D_{k}(\cdot, x)\right\|_{L^{1}(\mu)}^{1 / p}\left\|D_{j} D_{k}(\cdot, x)\right\|_{L^{\infty}(\mu)}^{1-1 / p} \\
& \leq C 2^{-2|j-k| \theta} \frac{1}{\ell\left(Q_{x, k+1}\right)^{(1-1 / p) n}} .
\end{aligned}
$$

Combining the estimates (3.1), (3.2) and (3.3) yields that

$$
\begin{aligned}
\left\|D_{k}(x, \cdot)\right\|_{\dot{B}_{p q}^{s}(\mu)} & =\left\{\sum_{j=-\infty}^{\infty} 2^{j s q}\left\|D_{j} D_{k}(\cdot, x)\right\|_{L^{p}(\mu)}^{q}\right\}^{1 / q} \\
& \leq C \frac{1}{\ell\left(Q_{x, k+1}\right)^{(1-1 / p) n}}\left\{\sum_{j=-\infty}^{\infty} 2^{j s q-2 \theta|j-k| q}\right\}^{1 / q} \\
& \leq C 2^{k s} \frac{1}{\ell\left(Q_{x, k+1}\right)^{(1-1 / p) n}}
\end{aligned}
$$

where $C$ is independent of $k$ and $x$. This proves the lemma.

Remark 3.1. More generally, we can verify that if $f \in C^{1}\left(\mathbb{R}^{d}\right)$ with compact support and

$$
\int_{\mathbb{R}^{d}} f(x) d \mu(x)=0
$$

then $f \in \dot{\mathcal{B}}_{p q}^{s}(\mu)$ for $|s|<\theta$ with $\theta$ the same as in Definition 2.5 and $1 \leq p, q \leq \infty$. We leave the details to the reader.

We can now introduce the Besov spaces $\dot{B}_{p q}^{s}(\mu)$.

Definition 3.1. Let $\theta$ be the same as in Definition 2.5 and let $|s|<\theta$. Let $p^{\prime}$ and $q^{\prime}$ be the conjugate index of $p$ and $q$, respectively, and let $\left\{D_{k}\right\}_{k=-\infty}^{\infty}$ be as in 
Theorem 2.1. For $1 \leq p, q \leq \infty$, we define

$$
\dot{B}_{p q}^{s}(\mu)=\left\{f \in\left(\dot{\mathcal{B}}_{p^{\prime}, q^{\prime}}^{-s}(\mu)\right)^{\prime}:\|f\|_{\dot{B}_{p q}^{s}(\mu)}<\infty\right\},
$$

where

$$
\|f\|_{\dot{B}_{p q}^{s}(\mu)}=\left\{\sum_{k=-\infty}^{\infty} 2^{k s q}\left\|D_{k} f\right\|_{L^{p}(\mu)}^{q}\right\}^{1 / q} .
$$

Based on Lemma 3.1 and Theorem 2.2, for all $f \in\left(\dot{\mathcal{B}}_{p^{\prime}, q^{\prime}}^{-s}(\mu)\right)^{\prime}$ with $1 \leq p, q \leq$ $\infty$, we have

$$
E_{j} f(x)=\sum_{k=-\infty}^{\infty} E_{j} D_{k}^{N} D_{k} T_{N}^{-1}(f)(x)
$$

where $\left\{P_{k}\right\}_{k \in \mathbb{Z}}$ is an approximation to the identity as in Definition 2.9, $E_{k}=$ $P_{k}-P_{k-1}$ for $k \in \mathbb{Z}$, and $N \in \mathbb{N}$ is large enough such that (2.45) holds. The above equality and the same proof of Proposition 2.1 show that spaces $\dot{B}_{p q}^{s}(\mu)$ are independent of the choice of approximations to the identity as in Definition 2.9. We leave these details to the reader.

It is well known that the space of Schwartz functions is dense in Besov spaces on $\mathbb{R}^{d}$. The following result shows that our space of test functions, $\dot{\mathcal{B}}_{p, q}^{s}(\mu)$, is also dense in the Besov space $\dot{B}_{p q}^{s}(\mu)$.

Proposition 3.1. Let $\theta$ be the same as in Definition 2.5, $|s|<\theta$ and $1 \leq p, q \leq \infty$. Then

$$
\overline{\dot{\mathcal{B}}_{p q}^{s}(\mu)}=\dot{B}_{p q}^{s}(\mu)
$$

where $\overline{\dot{\mathcal{B}}_{p q}^{s}(\mu)}$ is the closure of $\dot{\mathcal{B}}_{p q}^{s}(\mu)$ with respect to the norm $\|\cdot\|_{\dot{B}_{p q}^{s}(\mu)}$.

Proof. We first show $\overline{\dot{\mathcal{B}}_{p q}^{s}(\mu)} \subset \dot{B}_{p q}^{s}(\mu)$. To do this, we claim that if $1 \leq p, q \leq \infty$ and $f \in \dot{\mathcal{B}}_{p q}^{s}(\mu)$, then $f \in\left(\dot{\mathcal{B}}_{p^{\prime}, q^{\prime}}^{-s}(\mu)\right)^{\prime}$ and

$$
\|f\|_{\left(\dot{\mathcal{B}}_{p^{\prime}, q^{\prime}}^{-s}(\mu)\right)^{\prime}} \leq C\|f\|_{\dot{B}_{p q}^{s}(\mu)} .
$$

Moreover, let $\left\{f_{k}\right\}_{k \in \mathbb{N}}$ be a Cauchy sequence of $\dot{\mathcal{B}}_{p q}^{s}(\mu)$ according to the norm $\|\cdot\|_{\dot{B}_{p q}^{s}(\mu)}$. Then there is an $f \in\left(\dot{\mathcal{B}}_{p^{\prime}, q^{\prime}}^{-s}(\mu)\right)^{\prime}$ such that $\|f\|_{\dot{B}_{p q}^{s}(\mu)}<\infty$ and $f_{k} \rightarrow f$ in $\dot{B}_{p q}^{s}(\mu)$ as $k \rightarrow \infty$.

To show the above claim, let $f \in \dot{\mathcal{B}}_{p q}^{s}(\mu)$ and $g \in \dot{\mathcal{B}}_{p^{\prime}, q^{\prime}}^{-s}(\mu)$. Let $\left\{D_{k}\right\}_{k \in \mathbb{Z}}$ be the same as in Theorem 2.1 and recall $D_{k}=S_{k}-S_{k-1}$ for $k \in \mathbb{Z}$. It is easy to see that $D_{k}^{N}$ for $k \in \mathbb{Z}$ has the same properties as those of $D_{k}$ for $k \in \mathbb{Z}$, deduced from Lemma 2.4, with a constant depending on $N$, namely $C N$, if $C$ is the constant appearing in the properties satisfied by $D_{k}$ for $k \in \mathbb{Z}$; see Lemma 2.4. 
Noting $\left(D_{k}^{N}\right)^{*}=D_{k}^{N}$, by (2.37), the Hölder inequality, Theorem 2.1 and Proposition 2.1 we obtain

$$
\begin{aligned}
|f(g)| & \left.=|\langle f, g\rangle| \quad \text { in the sense of }\left(L^{2}(\mu)\right)^{\prime}=L^{2}(\mu)\right) \\
& =\left|\int_{\mathbb{R}^{d}} \sum_{k=-\infty}^{\infty} D_{k}^{N} D_{k} T_{N}^{-1}(f) g d \mu\right| \\
& =\left|\sum_{k=-\infty}^{\infty} \int_{\mathbb{R}^{d}} D_{k} T_{N}^{-1}(f) D_{k}^{N}(g) d \mu\right| \\
& \leq \sum_{k=-\infty}^{\infty}\left\|D_{k} T_{N}^{-1}(f)\right\|_{L^{p}(\mu)}\left\|D_{k}^{N}(g)\right\|_{L^{p^{\prime}}(\mu)} \\
& \leq\left\{\sum_{k=-\infty}^{\infty} 2^{k s q}\left\|D_{k} T_{N}^{-1}(f)\right\|_{L^{p}(\mu)}^{q}\right\}^{1 / q}\left\{\sum_{k=-\infty}^{\infty} 2^{-k s q^{\prime}}\left\|D_{k}^{N}(g)\right\|_{L^{p^{\prime}}(\mu)}^{q^{\prime}}\right\}^{1 / q^{\prime}} \\
& \leq C_{N}\left\|T_{N}^{-1}(f)\right\|_{\dot{B}_{p q}^{s}(\mu)}\|g\|_{\dot{B}_{p^{\prime}, q^{\prime}}^{-s}(\mu)} \\
& \leq C_{N}\|f\|_{\dot{B}_{p q}^{s}(\mu)}\|g\|_{\dot{B}_{p^{\prime}, q^{\prime}}^{-s}(\mu)},
\end{aligned}
$$

where $C_{N}>0$ depends on $N$. Thus, $f \in\left(\dot{\mathcal{B}}_{p^{\prime}, q^{\prime}}^{-s}(\mu)\right)^{\prime}$ and

$$
\|f\|_{\left(\dot{\mathcal{B}}_{p^{\prime}, q^{\prime}}^{-s}(\mu)\right)^{\prime}} \leq C\|f\|_{\dot{B}_{p q}^{s}(\mu)} \text {. }
$$

That is, (3.5) holds.

Now let $\left\{f_{k}\right\}_{k \in \mathbb{N}}$ be a Cauchy sequence of $\dot{\mathcal{B}}_{p q}^{s}(\mu)$ according to the norm $\|\cdot\|_{\dot{B}_{p q}^{s}(\mu)}$. Then, by (3.5), it is also a Cauchy sequence according to the norm $\|\cdot\|_{\left(\dot{\mathcal{B}}_{p^{\prime}, q^{\prime}}^{-s}(\mu)\right)^{\prime}}$. Since $\left(\dot{\mathcal{B}}_{p^{\prime}, q^{\prime}}^{-s}(\mu)\right)^{\prime}$ is a Banach space (see [33]), then there is an $f \in\left(\dot{\mathcal{B}}_{p^{\prime}, q^{\prime}}^{-s}(\mu)\right)^{\prime}$ such that $f_{k} \rightarrow f$ in $\left(\dot{\mathcal{B}}_{p^{\prime}, q^{\prime}}^{-s}(\mu)\right)^{\prime}$ as $k \rightarrow \infty$. We still need to verify that $\|f\|_{\dot{B}_{p q}^{s}(\mu)}<\infty$. From Lemma 3.1 and

$$
\left|D_{k}\left(f_{n}-f\right)(x)\right| \leq\left\|D_{k}(x, \cdot)\right\|_{\dot{B}_{p q}^{s}(\mu)}\left\|f_{n}-f\right\|_{\left(\dot{\mathcal{B}}_{p^{\prime}, q^{\prime}}^{-s}(\mu)\right)^{\prime}},
$$

it follows that for all $x \in \operatorname{supp}(\mu)$ and all $k \in \mathbb{Z}$,

$$
\lim _{n \rightarrow \infty} D_{k} f_{n}(x)=D_{k} f(x) \text {. }
$$

Thus, the fact that $\left\|f_{n}\right\|_{\dot{B}_{p q}^{s}(\mu)} \leq C$ with $C$ independent of $n$, Definition 3.1, the Fatou lemma and (3.6) tell us that

$$
\|f\|_{\dot{B}_{p q}^{s}(\mu)} \leq C
$$

which shows $f \in \dot{B}_{p q}^{s}(\mu)$ and $f_{k} \rightarrow f$ in $\dot{B}_{p q}^{s}(\mu)$ as $k \rightarrow \infty$.

We now prove the other direction: $\dot{B}_{p q}^{s}(\mu) \subset \overline{\dot{\mathcal{B}}_{p q}^{s}(\mu)}$. This fact comes from Theorem 2.2 and its proof. More precisely, if $f \in \dot{B}_{p q}^{s}(\mu)$, then by Theorem 2.2 and its proof, we can write (2.46) as

$$
f=\sum_{k \in \mathbb{Z}} D_{k} D_{k}^{N} T_{N}^{-1}(f),
$$

where the series converges in the norm of $\dot{B}_{p q}^{s}(\mu)$. 
As in the proof of Theorem 2.2, if we define $g_{k, M}(x)$ by

$$
g_{k, M}(x)=\int_{Q_{0, M}} D_{k}(x, y)\left(D_{k}^{N} T_{N}^{-1}\right)(f)(y) d \mu(y),
$$

then $g_{k, M}(x)$ belongs to $\dot{\mathcal{B}}_{p q}^{s}(\mu)$ by Remark 3.1, and using (3.7), we can further verify $f$ can be approximated by a finite sum of $g_{k, M}(x)$. We leave the details to the reader. This shows $\dot{B}_{p q}^{s}(\mu) \subset \overline{\dot{\mathcal{B}}_{p q}^{s}(\mu)}$, and we have completed the proof of Proposition 3.1.

We remark that as a consequence, Proposition 3.1 indicates that $\dot{B}_{p q}^{s}(\mu)$ is a Banach space for $1 \leq p, q \leq \infty$.

We now establish the boundedness of the Riesz operators defined via the approximation to the identity in the spaces $\dot{B}_{p q}^{s}(\mu)$, and then we show that the spaces $\dot{B}_{p q}^{s}(\mu)$ have the lifting property by using these Riesz potential operators.

Definition 3.2. Let $\left\{D_{k}\right\}_{k \in \mathbb{Z}}$ be the same as in Theorem 2.1. For $\alpha \in \mathbb{R}, f \in L^{2}(\mu)$ and all $x \in \operatorname{supp}(\mu)$, we define the Riesz potential operator $I_{\alpha}$ by

$$
I_{\alpha} f(x)=\sum_{k=-\infty}^{\infty} 2^{-k \alpha} D_{k} f(x) .
$$

Theorem 3.1. Let $\theta$ be the same as in Definition 2.5, let $|s|<\theta$ and let $|s+\alpha|<\theta$. Then $I_{\alpha}$ is bounded from $\dot{B}_{p q}^{s}(\mu)$ to $\dot{B}_{p q}^{s+\alpha}(\mu)$ for $1 \leq p, q \leq \infty$, namely, there is a constant $C>0$ such that for all $f \in \dot{B}_{p q}^{s}(\mu)$ with $s, p$ and $q$ as above,

$$
\left\|I_{\alpha} f\right\|_{\dot{B}_{p q}^{s+\alpha}(\mu)} \leq C\|f\|_{\dot{B}_{p q}^{s}(\mu)} .
$$

Proof. Let $\left\{D_{k}\right\}_{k \in \mathbb{Z}}$ be the same as in Theorem 2.1. From Theorem 2.2 and the Minkowski inequality, it follows that

$$
\begin{aligned}
\left\|I_{\alpha} f\right\|_{\dot{B}_{p q}^{s+\alpha}(\mu)}^{s+\alpha} & =\left\{\sum_{j=-\infty}^{\infty} 2^{j(s+\alpha) q}\left\|D_{j} I_{\alpha} f\right\|_{L^{p}(\mu)}^{q}\right\}^{1 / q} \\
& \leq\left\{\sum_{j=-\infty}^{\infty} 2^{j(s+\alpha) q}\left[\sum_{k=-\infty}^{\infty}\left\|D_{j} I_{\alpha} D_{k}^{N} D_{k} T_{N}^{-1} f\right\|_{L^{p}(\mu)}\right]^{q}\right\}^{1 / q} \\
& \leq\left\{\sum_{j=-\infty}^{\infty} 2^{j(s+\alpha) q}\left[\sum_{k=-\infty}^{\infty} \sum_{i=-\infty}^{\infty} 2^{-i \alpha}\left\|D_{j} D_{i} D_{k}^{N} D_{k} T_{N}^{-1} f\right\|_{L^{p}(\mu)}\right]^{q}\right\}^{1 / q},
\end{aligned}
$$

where we assume that $N$ satisfies $(2.31)$.

Since $|s|<\theta$ and $|s+\alpha|<\theta$, we can choose $\nu \in(0,1 / 2)$ such that $|s+\alpha|<2 \nu \theta$, $|s|<2 \nu \theta$ and $|s|<2(1-\nu) \theta$. Similar to (2.41) and (2.42), by Lemma 2.5, we have

$$
\left\|D_{j} D_{i} D_{k}^{N} D_{k} T_{N}^{-1} f\right\|_{L^{p}(\mu)} \leq C 2^{-2 \theta|j-i|}\left\|D_{k} T_{N}^{-1} f\right\|_{L^{p}(\mu)}
$$

and

$$
\left\|D_{j} D_{i} D_{k}^{N} D_{k} T_{N}^{-1} f\right\|_{L^{p}(\mu)} \leq C 2^{-2 \theta|i-k|}\left\|D_{k} T_{N}^{-1} f\right\|_{L^{p}(\mu)} .
$$

The geometric means of (3.9) and (3.10) tells us that

$$
\left\|D_{j} D_{i} D_{k}^{N} D_{k} T_{N}^{-1} f\right\|_{L^{p}(\mu)} \leq C 2^{-2 \theta \nu|j-i|} 2^{-2 \theta(1-\nu)|i-k|}\left\|D_{k} T_{N}^{-1} f\right\|_{L^{p}(\mu)} .
$$


Inserting (3.11) into (3.8), the Hölder inequality and Theorem 2.1 yield that

$$
\begin{aligned}
& \left\|I_{\alpha} f\right\|_{\dot{B}_{p q}^{s+\alpha}(\mu)} \leq C\left\{\sum _ { j = - \infty } ^ { \infty } \left[\sum_{i=-\infty}^{\infty} 2^{(j-i)(s+\alpha)-2 \theta \nu|j-i|}\right.\right. \\
& \left.\left.\times \sum_{k=-\infty}^{\infty} 2^{i s} 2^{-2 \theta(1-\nu)|i-k|}\left\|D_{k} T_{N}^{-1} f\right\|_{L^{p}(\mu)}\right]^{q}\right\}^{1 / q} \\
& \leq C\left\{\sum _ { j = - \infty } ^ { \infty } \left[\left(\sum_{i=-\infty}^{\infty} 2^{(j-i)(s+\alpha)-2 \theta \nu|j-i|}\right.\right.\right. \\
& \left.\times\left[\sum_{k=-\infty}^{\infty} 2^{i s} 2^{-2 \theta(1-\nu)|i-k|}\left\|D_{k} T_{N}^{-1} f\right\|_{L^{p}(\mu)}\right]^{q}\right)^{1 / q} \\
& \left.\left.\times\left(\sum_{i=-\infty}^{\infty} 2^{(j-i)(s+\alpha)-2 \theta \nu|j-i|}\right)^{1 / q^{\prime}}\right]^{q}\right\}^{1 / q} \\
& \leq C\left\{\sum_{i=-\infty}^{\infty}\left[\sum_{k=-\infty}^{\infty} 2^{(i-k) s-2 \theta(1-\nu)|i-k|} 2^{k s}\left\|D_{k} T_{N}^{-1} f\right\|_{L^{p}(\mu)}\right]^{q}\right\}^{1 / q} \\
& \leq C\left\{\sum _ { i = - \infty } ^ { \infty } \left[\left(\sum_{k=-\infty}^{\infty} 2^{(i-k) s-2 \theta(1-\nu)|i-k|} 2^{k s q}\left\|D_{k} T_{N}^{-1} f\right\|_{L^{p}(\mu)}^{q}\right)^{1 / q}\right.\right. \\
& \left.\left.\times\left(\sum_{k=-\infty}^{\infty} 2^{(i-k) s-2 \theta(1-\nu)|i-k|}\right)^{1 / q^{\prime}}\right]^{q}\right\}^{1 / q} \\
& \leq C\left\{\sum_{k=-\infty}^{\infty} 2^{k s q}\left\|D_{k} T_{N}^{-1} f\right\|_{L^{p}(\mu)}^{q}\right\}^{1 / q} \\
& =C\left\|T_{N}^{-1} f\right\|_{\dot{B}_{p q}^{s}(\mu)} \\
& \leq C\|f\|_{\dot{B}_{p q}^{s}(\mu)},
\end{aligned}
$$

where $1 / q+1 / q^{\prime}=1$. This proves that $I_{\alpha}$ is bounded from $\dot{B}_{p q}^{s}(\mu)$ to $\dot{B}_{p q}^{s+\alpha}(\mu)$ and we have completed the proof of Theorem 3.1 .

We now establish the converse of Theorem 3.1. To this end, we will first show that when $\alpha$ is very small, the composition operator $I_{\alpha} I_{-\alpha}$ is invertible in the spaces $\dot{B}_{p q}^{s}(\mu)$. To do so, for any given $N_{1} \in \mathbb{N}$, we decompose $I-I_{\alpha} I_{-\alpha}$ into

$$
\begin{aligned}
I-I_{\alpha} I_{-\alpha}= & \sum_{i=-\infty}^{\infty} \sum_{|m| \leq N_{1}}\left(1-2^{m \alpha}\right) D_{i} D_{i+m} \\
& +\sum_{i=-\infty}^{\infty} \sum_{|m|>N_{1}}\left(1-2^{m \alpha}\right) D_{i} D_{i+m} \\
= & L_{N_{1}}^{1}+L_{N_{1}}^{2} .
\end{aligned}
$$


We will show that if $N_{1}$ is large enough and if $\alpha$ is small enough, then the operator norms of $L_{N_{1}}^{i}$ in the spaces $\dot{B}_{p q}^{s}(\mu)$ will be very small for $i=1,2$. Thus, $I_{\alpha} I_{-\alpha}$ is invertible in the spaces $\dot{B}_{p q}^{s}(\mu)$.

Theorem 3.2. Let $\theta$ be the same as in Definition 2.5, let $|s|<\theta$ and let $|s-\alpha|<\theta$. Then for any $\nu \in(0,1 / 2)$ such that $|s|<2 \nu \theta$ and $|s-\alpha|<2 \nu \theta$,

$$
\left\|L_{N_{1}}^{1}\right\|_{\dot{B}_{p q}^{s}(\mu) \rightarrow \dot{B}_{p q}^{s}(\mu)} \leq C_{5} \sum_{|m| \leq N_{1}}\left|1-2^{m \alpha}\right| 2^{-2 \theta \nu|m|-m s}
$$

and

$$
\left\|L_{N_{1}}^{2}\right\|_{\dot{B}_{p q}^{s}(\mu) \rightarrow \dot{B}_{p q}^{s}(\mu)} \leq C_{5} \sum_{|m|>N_{1}}\left|1-2^{m \alpha}\right| 2^{-2 \theta \nu|m|-m s}
$$

for $1 \leq p, q \leq \infty$. Here $C_{5}$ is independent of $N_{1}$ and $\alpha$.

Proof. We only show (3.12). The proof of (3.13) is similar.

To show (3.12), let $\left\{D_{k}\right\}_{k \in \mathbb{Z}}$ be the same as in Theorem 2.1. By Theorem 2.2 , for any $j \in \mathbb{Z}$, we can write

$$
D_{j} L_{N_{1}}^{1} f(x)=\sum_{i=-\infty}^{\infty} \sum_{k=-\infty}^{\infty} \sum_{|m| \leq N_{1}}\left(1-2^{m \alpha}\right) D_{j} D_{i} D_{i+m} D_{k}^{N} D_{k} T_{N}^{-1} f(x),
$$

where $N$ is as in (2.31).

Let $\nu$ be the same as in Theorem 3.2 Similar to (3.9) and (3.10), by Lemma 2.5, we have

$$
\begin{aligned}
& \left\|D_{j} D_{i} D_{i+m} D_{k}^{N} D_{k} T_{N}^{-1} f\right\|_{L^{p}(\mu)} \\
& \leq C N 2^{-2 \theta[|j-i|+|i+m-k|]}\left\|D_{k} T_{N}^{-1} f\right\|_{L^{p}(\mu)}
\end{aligned}
$$

and

$$
\left\|D_{j} D_{i} D_{i+m} D_{k}^{N} D_{k} T_{N}^{-1} f\right\|_{L^{p}(\mu)} \leq C N 2^{-2 \theta|m|}\left\|D_{k} T_{N}^{-1} f\right\|_{L^{p}(\mu)} .
$$

The geometric means of (3.15) and (3.16) implies that

$$
\begin{aligned}
& \left\|D_{j} D_{i} D_{i+m} D_{k}^{N} D_{k} T_{N}^{-1} f\right\|_{L^{p}(\mu)} \\
& \leq C N 2^{-2 \theta(1-\nu)[|j-i|+|i+m-k|]} 2^{-2 \theta \nu|m|}\left\|D_{k} T_{N}^{-1} f\right\|_{L^{p}(\mu)} .
\end{aligned}
$$


The formula (3.14), the estimate (3.17), the Minkowski inequality, the Hölder inequality and Theorem 2.1 tell us that

$$
\begin{aligned}
& \left\|L_{N_{1}}^{1} f\right\|_{\dot{B}_{p q}^{s}(\mu)} \\
& =\left\{\sum_{j=-\infty}^{\infty} 2^{j s q}\left\|D_{j} L_{N_{1}}^{1} f\right\|_{L^{p}(\mu)}^{q}\right\}^{1 / q} \\
& \leq C \sum_{|m| \leq N_{1}}\left|1-2^{m \alpha}\right| 2^{-2 \theta \nu|m|}\left\{\sum _ { j = - \infty } ^ { \infty } \left[\sum_{i=-\infty}^{\infty} 2^{(j-i) s-2 \theta(1-\nu)|j-i|}\right.\right. \\
& \left.\left.\times \sum_{k=-\infty}^{\infty} 2^{i s} 2^{-2 \theta(1-\nu)|i+m-k|]}\left\|D_{k} T_{N}^{-1} f\right\|_{L^{p}(\mu)}\right]^{q}\right\}^{1 / q} \\
& \leq C \sum_{|m| \leq N_{1}}\left|1-2^{m \alpha}\right| 2^{-2 \theta \nu|m|-m s} \\
& \times\left\{\sum_{i=-\infty}^{\infty}\left[\sum_{k=-\infty}^{\infty} 2^{(i+m-k) s-2 \theta(1-\nu)|i+m-k|]} 2^{k s}\left\|D_{k} T_{N}^{-1} f\right\|_{L^{p}(\mu)}\right]^{q}\right\}^{1 / q} \\
& \leq C \sum_{|m| \leq N_{1}}\left|1-2^{m \alpha}\right| 2^{-2 \theta \nu|m|-m s}\left\{\sum_{k=-\infty}^{\infty} 2^{k s q}\left\|D_{k} T_{N}^{-1} f\right\|_{L^{p}(\mu)}^{q}\right\}^{1 / q} \\
& \leq C \sum_{|m| \leq N_{1}}\left|1-2^{m \alpha}\right| 2^{-2 \theta \nu|m|-m s}\left\|T_{N}^{-1} f\right\|_{\dot{B}_{p q}^{s}(\mu)} \\
& \leq C \sum_{|m| \leq N_{1}}\left|1-2^{m \alpha}\right| 2^{-2 \theta \nu|m|-m s}\|f\|_{\dot{B}_{p q}^{s}(\mu)} .
\end{aligned}
$$

That is, (3.12) holds and we have finished the proof of Theorem 3.2.

From Theorem 3.2 it is easy to deduce the following result.

Corollary 3.1. Let $\theta$ be the same as in Definition 2.5, let $|s|<\theta$ and let $|s-\alpha|<\theta$. Then there is $\alpha_{0}(s)>0$ such that if $|\alpha|<\alpha_{0}(s)$ and $N_{1} \in \mathbb{N}$ is large enough,

$$
C_{5}\left\{\sum_{|m| \leq N_{1}}\left|1-2^{m \alpha}\right| 2^{-2 \theta \nu|m|-m s}+\sum_{|m|>N_{1}}\left|1-2^{m \alpha}\right| 2^{-2 \theta \nu|m|-m s}\right\}<1,
$$

where $\nu \in(0,1 / 2)$ satisfies that $|s|<2 \nu \theta$ and $|s-\alpha|<2 \nu \theta$. Thus, if $1 \leq p, q \leq \infty$ and $|\alpha|<\alpha_{0}(s)$, then $\left(I_{\alpha} I_{-\alpha}\right)^{-1}$ exists in $\dot{B}_{p q}^{s}(\mu)$ and

$$
\left\|\left(I_{\alpha} I_{-\alpha}\right)^{-1}\right\|_{\dot{B}_{p q}^{s}(\mu) \rightarrow \dot{B}_{p q}^{s}(\mu)} \leq C .
$$

If we change the order of $I_{\alpha}$ and $I_{-\alpha}$, we have a similar result which is a simple corollary of Corollary 3.1 above.

Corollary 3.2. Let $\theta$ be the same as in Definition 2.5, let $|s|<\theta$ and let $|s+\alpha|<\theta$. Then there is $\alpha_{0}(s)>0$ such that if $|\alpha|<\alpha_{0}(s)$ and $N_{1} \in \mathbb{N}$ is large enough,

$$
C_{5}\left\{\sum_{|m| \leq N_{1}}\left|1-2^{-m \alpha}\right| 2^{-2 \theta \nu|m|-m s}+\sum_{|m|>N_{1}}\left|1-2^{-m \alpha}\right| 2^{-2 \theta \nu|m|-m s}\right\}<1,
$$


where $\nu \in(0,1 / 2)$ satisfies that $|s|<2 \nu \theta$ and $|s+\alpha|<2 \nu \theta$. Thus, if $1 \leq p, q \leq \infty$ and $|\alpha|<\alpha_{0}(s)$, then $\left(I_{-\alpha} I_{\alpha}\right)^{-1}$ exists in $\dot{B}_{p q}^{s}(\mu)$ and

$$
\left\|\left(I_{-\alpha} I_{\alpha}\right)^{-1}\right\|_{\dot{B}_{p q}^{s}(\mu) \rightarrow \dot{B}_{p q}^{s}(\mu)} \leq C .
$$

Theorem 3.1 and Corollary 3.2 imply the following lifting theorem for the spaces $\dot{B}_{p q}^{s}(\mu)$.

Theorem 3.3. Let $\theta$ be the same as in Definition 2.5. $|s|<\theta$ and $|s+\alpha|<\theta$. Let $\alpha_{0}(s)$ be the same as in Corollary 3.2 and $|\alpha|<\alpha_{0}(s)$. Then, if $1 \leq p, q \leq \infty$, there is a constant $C_{6}>0$ such that for all $f \in \dot{B}_{p q}^{s}(\mu)$,

$$
C_{6}^{-1}\|f\|_{\dot{B}_{p q}^{s}(\mu)} \leq\left\|I_{\alpha} f\right\|_{\dot{B}_{p q}^{s+\alpha}(\mu)} \leq C_{6}\|f\|_{\dot{B}_{p q}^{s}(\mu)} .
$$

Proof. To show the theorem, we only need to verify its left-hand inequality. In fact, by Corollary 3.2, we have

$$
\|f\|_{\dot{B}_{p q}^{s}(\mu)}=\left\|\left(I_{-\alpha} I_{\alpha}\right)^{-1} I_{-\alpha} I_{\alpha}\right\|_{\dot{B}_{p q}^{s}(\mu)} \leq C\left\|I_{-\alpha} I_{\alpha}\right\|_{\dot{B}_{p q}^{s}(\mu)} \leq C\left\|I_{\alpha} f\right\|_{\dot{B}_{p q}^{s+\alpha}(\mu)} .
$$

We have completed the proof of Theorem 3.3 .

Finally, in this section, we study the dual spaces of the spaces $\dot{B}_{p q}^{s}(\mu)$. To begin with, we establish the following lemma.

Lemma 3.2. Let $\theta$ be the same as in Definition 2.5, let $|s|<\theta$, and let $\left\{D_{k}\right\}_{k \in \mathbb{Z}}$ be the same as in Theorem 2.1. Suppose that $\left\{g_{k}\right\}_{k \in \mathbb{Z}}$ is a sequence of functions on $\mathbb{R}^{d}$. If $1 \leq p, q<\infty$ and

$$
\left\{\sum_{k \in \mathbb{Z}} 2^{k s q}\left\|g_{k}\right\|_{L^{p}(\mu)}^{q}\right\}^{1 / q}<\infty,
$$

then $g(x)=\sum_{k \in \mathbb{Z}} D_{k} g_{k}(x) \in \dot{B}_{p q}^{s}(\mu)$ and

$$
\|g\|_{\dot{B}_{p q}^{s}(\mu)} \leq C\left\{\sum_{k=-\infty}^{\infty} 2^{k s q}\left\|g_{k}\right\|_{L^{p}(\mu)}^{q}\right\}^{1 / q}
$$

where $C$ is a positive constant.

Proof. For $L_{1}, L_{2} \in \mathbb{Z}$ and $L_{1}<L_{2}$, we define

$$
g_{L_{1}}^{L_{2}}(x)=\sum_{k=L_{1}}^{L_{2}} D_{k} g_{k}(x)
$$


Then for $f \in \dot{\mathcal{B}}_{p^{\prime}, q^{\prime}}^{-s}(\mu)$, noting that $D_{k}(x, y)=D_{k}(y, x)$ and by the Hölder inequality, we have

$$
\begin{aligned}
\left|\left\langle g_{L_{1}}^{L_{2}}, f\right\rangle\right| & =\left|\sum_{k=L_{1}}^{L_{2}}\left\langle D_{k} g_{k}, f\right\rangle\right| \\
& \leq \sum_{k=L_{1}}^{L_{2}}\left|\left\langle g_{k}, D_{k} f\right\rangle\right| \\
& \leq \sum_{k=L_{1}}^{L_{2}}\left\|g_{k}\right\|_{L^{p}(\mu)}\left\|D_{k} f\right\|_{L^{p^{\prime}}(\mu)} \\
& \leq\left\{\sum_{k=L_{1}}^{L_{2}} 2^{k s q}\left\|g_{k}\right\|_{L^{p}(\mu)}^{q}\right\}^{1 / q}\left\{\sum_{k=L_{1}}^{L_{2}} 2^{-k s q^{\prime}}\left\|D_{k} f\right\|_{L^{p^{\prime}}(\mu)}^{q^{\prime}}\right\}^{1 / q^{\prime}} \\
& \leq\left\{\sum_{k=L_{1}}^{L_{2}} 2^{k s q}\left\|g_{k}\right\|_{L^{p}(\mu)}^{q}\right\}^{1 / q}\|f\|_{\dot{\mathcal{B}}_{p^{\prime}, q^{\prime}}^{-s}(\mu)} .
\end{aligned}
$$

Thus, $g_{L_{1}}^{L_{2}} \in\left(\dot{\mathcal{B}}_{p^{\prime}, q^{\prime}}^{-s}(\mu)\right)^{\prime}$ and

$$
\left\|g_{L_{1}}^{L_{2}}\right\|_{\left(\dot{\mathcal{B}}_{p^{\prime}, q^{\prime}}^{-s}(\mu)\right)^{\prime}} \leq\left\{\sum_{k=L_{1}}^{L_{2}} 2^{k s q}\left\|g_{k}\right\|_{L^{p}(\mu)}^{q}\right\}^{1 / q} .
$$

From this, it follows that $g \in\left(\dot{\mathcal{B}}_{p^{\prime}, q^{\prime}}^{-s}(\mu)\right)^{\prime}$, and Lemma 2.5 and the Hölder inequality now tell us that

$$
\begin{aligned}
\|g\|_{\dot{B}_{p q}^{s}(\mu)} & \left\{\sum_{j=-\infty}^{\infty} 2^{j s q}\left\|D_{j} g\right\|_{L^{p}(\mu)}^{q}\right\}^{1 / q} \\
\leq & \left\{\sum_{j=-\infty}^{\infty} 2^{j s q}\left[\sum_{k=-\infty}^{\infty}\left\|D_{j} D_{k} g_{k}\right\|_{L^{p}(\mu)}\right]^{q}\right\}^{1 / q} \\
\leq & C\left\{\sum_{j=-\infty}^{\infty}\left[\sum_{k=-\infty}^{\infty} 2^{(j-k) s-2|j-k| \theta} 2^{k s}\left\|g_{k}\right\|_{L^{p}(\mu)}\right]^{q}\right\}^{1 / q} \\
\leq & C\left\{\sum _ { j = - \infty } ^ { \infty } \left[\left(\sum_{k=-\infty}^{\infty} 2^{(j-k) s-2|j-k| \theta} 2^{k s q}\left\|g_{k}\right\|_{L^{p}(\mu)}^{q}\right)^{1 / q}\right.\right. \\
& \left.\left.\times\left(\sum_{k=-\infty}^{\infty} 2^{(j-k) s-2|j-k| \theta}\right)^{1 / q^{\prime}}\right]^{q}\right\}^{1 / q} \\
\leq & C\left\{\sum_{k=-\infty}^{\infty}\left[\sum_{j=-\infty}^{\infty} 2^{(j-k) s-2|j-k| \theta}\right]^{k s q}\left\|g_{k}\right\|_{L^{p}(\mu)}^{q}\right\}^{1 / q} \\
\leq & C\left\{\sum_{k=-\infty}^{\infty} 2^{k s q}\left\|g_{k}\right\|_{L^{p}(\mu)}^{q}\right\}^{1 / q} \cdot
\end{aligned}
$$

That is, $g \in \dot{B}_{p q}^{s}(\mu)$ and we have finished the proof of Lemma 3.2 . 
We now can establish the dual theorem for the spaces $\dot{B}_{p q}^{s}(\mu)$.

Theorem 3.4. Let $\theta$ be the same as in Definition 2.5 and let $|s|<\theta$. If $1 \leq p, q \leq$ $\infty$ and $g \in \dot{B}_{p q}^{s}(\mu)$, then

$$
\mathcal{L}_{g}(f)=\langle g, f\rangle
$$

defines a linear functional on $\dot{\mathcal{B}}_{p^{\prime}, q^{\prime}}^{-s}(\mu)$ and

$$
\left\|\mathcal{L}_{g}\right\|_{\left(\dot{\mathcal{B}}_{p^{\prime}, q^{\prime}}^{-s}(\mu)\right)^{\prime}} \leq C\|g\|_{\dot{B}_{p q}^{s}(\mu)} .
$$

Conversely, if $1<p, q<\infty$ and $\mathcal{L}$ is a linear functional on $\dot{B}_{p q}^{s}(\mu)$, then there exists a unique $g \in \dot{B}_{p^{\prime}, q^{\prime}}^{-s}(\mu)$ such that

$$
\mathcal{L}(f)=\langle g, f\rangle
$$

on $\dot{\mathcal{B}}_{p q}^{s}(\mu)$ and

$$
\|g\|_{\dot{B}_{p^{\prime}, q^{\prime}}^{-s}(\mu)} \leq C\|\mathcal{L}\|_{\left(\dot{B}_{p q}^{s}(\mu)\right)^{\prime}} .
$$

Proof. The estimate (3.18) is just (3.5) in Proposition 3.1

Conversely, suppose that $\mathcal{L}$ is a linear functional on $\dot{B}_{p q}^{s}(\mu)$. By Proposition 3.1 . it is easy to see that $\mathcal{L}$ is also a linear functional on $\dot{\mathcal{B}}_{p q}^{s}(\mu)$, and therefore, for all $f \in \dot{\mathcal{B}}_{p q}^{s}(\mu)$,

$$
|\mathcal{L}(f)| \leq\|\mathcal{L}\|_{\left(\dot{B}_{p q}^{s}(\mu)\right)^{\prime}}\|f\|_{\dot{B}_{p q}^{s}(\mu)} .
$$

Let $\left\{D_{k}\right\}_{k \in \mathbb{Z}}$ be the same as in Theorem 2.1. If $f \in \dot{\mathcal{B}}_{p q}^{s}(\mu)$, then the sequence $\left\{D_{k} f\right\}_{k \in \mathbb{Z}}$ is in the sequence space

$$
l_{q}^{s}\left(L^{p}\right)=\left\{\left\{f_{k}\right\}_{k \in \mathbb{Z}}:\left\|\left\{f_{k}\right\}_{k \in \mathbb{Z}}\right\|_{l_{q}^{s}\left(L^{p}\right)}=\left\{\sum_{k=-\infty}^{\infty} 2^{k s q}\left\|f_{k}\right\|_{L^{p}(\mu)}^{q}\right\}^{1 / q}<\infty\right\} .
$$

Define $\widetilde{\mathcal{L}}$ on a subset of the sequence space $l_{q}^{s}\left(L^{p}\right)$ by

$$
\widetilde{\mathcal{L}}\left[\left\{D_{k} f\right\}_{k \in \mathbb{Z}}\right]=\mathcal{L}(f) .
$$

Then, if $f \in \dot{\mathcal{B}}_{p q}^{s}(\mu)$, we have

$$
\begin{aligned}
\left|\widetilde{\mathcal{L}}\left[\left\{D_{k} f\right\}_{k \in \mathbb{Z}}\right]\right| & =|\mathcal{L}(f)| \leq\|\mathcal{L}\|_{\left(\dot{B}_{p q}^{s}(\mu)\right)^{\prime}}\|f\|_{\dot{B}_{p q}^{s}(\mu)} \\
& =\|\mathcal{L}\|_{\left(\dot{B}_{p q}^{s}(\mu)\right)^{\prime}}\left\|\left\{D_{k} f\right\}_{k \in \mathbb{Z}}\right\|_{l_{q}^{s}\left(L^{p}\right)} .
\end{aligned}
$$

Thus, $\widetilde{\mathcal{L}}$ is bounded on this subset. The Hahn-Banach theorem tells us that $\widetilde{\mathcal{L}}$ can be extended to a functional on $l_{q}^{s}\left(L^{p}\right)$. Since it is well known that $\left(l_{q}^{s}\left(L^{p}\right)\right)^{\prime}=l_{q^{\prime}}^{-s}\left(L^{p^{\prime}}\right)$ for $1 \leq p, q<\infty$ (see [30]), there exists a unique sequence $\left\{g_{k}\right\}_{k \in \mathbb{Z}} \in l_{q^{\prime}}^{-s}\left(L^{p^{\prime}}\right)$ such that

$$
\left\|\left\{g_{k}\right\}_{k \in \mathbb{Z}}\right\|_{l_{q^{\prime}}^{-s}\left(L^{p^{\prime}}\right)} \leq C\|\widetilde{\mathcal{L}}\|_{\left(l_{q}^{s}\left(L^{p}\right)\right)^{\prime}} \leq C\|\mathcal{L}\|_{\left(\dot{B}_{p q}^{s}(\mu)\right)^{\prime}}
$$

and

$$
\widetilde{\mathcal{L}}\left[\left\{f_{k}\right\}_{k \in \mathbb{Z}}\right]=\sum_{k=-\infty}^{\infty}\left\langle g_{k}, f_{k}\right\rangle
$$


for all $\left\{f_{k}\right\}_{k \in \mathbb{Z}} \in l_{q}^{s}\left(L^{p}\right)$. Thus, if $f \in \dot{\mathcal{B}}_{p q}^{s}(\mu)$, then Lemma 3.2 yields that

$$
\begin{aligned}
\mathcal{L}(f) & =\widetilde{\mathcal{L}}\left(\left\{D_{k} f\right\}_{k \in \mathbb{Z}}\right)=\sum_{k=-\infty}^{\infty}\left\langle g_{k}, D_{k}(f)\right\rangle \\
& =\sum_{k=-\infty}^{\infty}\left\langle D_{k}\left(g_{k}\right), f\right\rangle=\left\langle\sum_{k=-\infty}^{\infty} D_{k}\left(g_{k}\right), f\right\rangle,
\end{aligned}
$$

since $D_{k}^{*}=D_{k}$. Let

$$
g=\sum_{k=-\infty}^{\infty} D_{k}\left(g_{k}\right)
$$

Then Lemma 3.2 tells us that $g \in \dot{B}_{p^{\prime}, q^{\prime}}^{-s}(\mu)$ and

$$
\|g\|_{\dot{B}_{p^{\prime}, q^{\prime}}^{-s}(\mu)} \leq C\left\|\left\{g_{k}\right\}_{k \in \mathbb{Z}}\right\|_{l_{q^{\prime}}^{-s}\left(L^{p^{\prime}}\right)} \leq C\|\mathcal{L}\|_{\left(\dot{B}_{p q}^{s}(\mu)\right)^{\prime}} .
$$

Thus, (3.19) holds.

This finishes the proof of Theorem 3.4

\section{ACKNOWLEDGMENT}

The authors thank the referee for his/her very careful reading and many valuable remarks which made this article more readable.

\section{REFERENCES}

[1] A. P. Calderón, Intermediate spaces and interpolation, the complex method, Studia Math. 24 (1964), 113-190. MR0167830 (29:5097)

[2] R. R. Coifman and G. Weiss, Analyse Harmonique Non-commutative sur Certains Espaces Homogènes, Lecture Notes in Math. 242, Springer-Verlag, Berlin, 1971. MR0499948 $(58: 17690)$

[3] G. David, J. L. Journé and S. Semmes, Opérateurs de Calderón-Zygmund, fonctions paraaccrétives et interpolation, Rev. Mat. Iberoam. 1 (1985), 1-56. MR0850408 (88f:47024)

[4] M. Frazier, B. Jawerth and G. Weiss, Littlewood-Paley theory and the study of function spaces, CBMS Regional Conference Series in Math. 79, Amer. Math. Soc. Providence, R. I., 1991. MR.1107300 (92m:42021)

[5] J. García-Cuerva and A. E. Gatto, Boundedness properties of fractional integral operators associated to non-doubling measures, Studia Math. 162 (2004), 245-261. MR2047654

[6] J. García-Cuerva and A. E. Gatto, Lipschitz spaces and Calderón-Zygmund operators associated to non-doubling measures, http://arxiv.org/math/0212289.

[7] J. García-Cuerva and J.M. Martell, Weighted inequalities and vector-valued CalderónZygmund operators on non-homogeneous spaces, Publ. Mat. 44 (2000), 613-640. MR.1800824 (2002k:42031)

[8] J. García-Cuerva and J. M. Martell, On the existence of principal values for the Cauchy integral on weighted Lebesgue spaces for non-doubling measures, J. Fourier Anal. Appl. 7 (2001), 469-487. MR1845099 (2002i:42013)

[9] J. E. Gilbert, Y. Han, J. A. Hogan, J. D. Lakey, D. Weiland and G. Weiss, Smooth molecular decompositions of functions and singular integral operators, Mem. Amer. Math. Soc. 156 (2002), No. 742, 1-74. MR1880991 (2003f:42026)

[10] Y. Han, Calderón-type reproducing formula and the Tb theorem, Rev. Mat. Iberoam. 10 (1994), 51-91. MR1271757 (95h:42020)

[11] Y. Han, Plancherel-Pólya type inequality on spaces of homogeneous type and its applications, Proc. Amer. Math. Soc. 126 (1998), 3315-3327. MR1459123 (99a:42010)

[12] Y. Han and E. T. Sawyer, Littlewood-Paley theory on spaces of homogeneous type and classical function spaces, Mem. Amer. Math. Soc. 110 (1994), No. 530, 1-126. MR1214968 (96a:42016) 
[13] Y. Han and D. Yang, New characterizations and applications of inhomogeneous Besov and Triebel-Lizorkin spaces on homogeneous type spaces and fractals, Dissertationes Math. (Rozprawy Mat.) 403 (2002), 1-102. MR1926534 (2003h:46051)

[14] Y. Han and D. Yang, Some new spaces of Besov and Triebel-Lizorkin type on homogeneous spaces, Studia Math. 156 (2003), 67-97. MR 1961062 (2004a:42018)

[15] J. Mateu, P. Mattila, A. Nicolau and J. Orobitg, BMO for nondoubling measures, Duke Math. J. 102 (2000), 533-565. MR1756109 (2001e:26019)

[16] F. Nazarov, S. Treil and A. Volberg, Cauchy integral and Calderón-Zygmund operators on nonhomogeneous spaces, Internat. Math. Res. Notices 1997, no. 15, 703-726. MR1470373 (99e:42028)

[17] F. Nazarov, S. Treil and A. Volberg, Weak type estimates and Cotlar inequalities for Calderón-Zygmund operators on nonhomogeneous spaces, Internat. Math. Res. Notices 1998, no. 9, 463-487. MR1626935 (99f:42035)

[18] F. Nazarov, S. Treil and A. Volberg, The Tb-theorem on nonhomogeneous spaces, Acta Math. 190 (2003), 151-239. MR.1998349

[19] F. Nazarov, S. Treil and A. Volberg, Accretive system Tb-theorems on nonhomogeneous spaces, Duke Math. J. 113 (2002), 259-312. MR.1909219 (2003g:42030)

[20] J. Orobitg and C. Pérez, $A_{p}$ weights for nondoubling measures in $\mathbb{R}^{n}$ and applications, Trans. Amer. Math. Soc. 354 (2002), 2013-2033. MR1881028 (2002k:42044)

[21] J. Peetre, New Thoughts on Besov Spaces, Duke Univ. Math. Series, No. 1., Durham, N. C., 1976. MR0461123 (57:1108)

[22] E. M. Stein, Harmonic Analysis: Real-variable Methods, Orthogonality, and Oscillatory Integrals, Princeton Univ. Press, Princeton, N. J., 1993. MR.1232192 (95c:42002)

[23] X. Tolsa, Cotlar's inequality without the doubling condition and existence of principal values for the Cauchy integral of measures, J. Reine Angew. Math. 502 (1998), 199-235. MR 1647575 (2000a:42030)

[24] X. Tolsa, $L^{2}$-boundedness of the Cauchy integral operator for continuous measures, Duke Math. J. 98 (1999), 269-304. MR1695200(2000d:31001)

[25] X. Tolsa, A T (1) theorem for non-doubling measures with atoms, Proc. London Math. Soc. (3) 82 (2001), 195-228. MR.1794262 (2002i:42019)

[26] X. Tolsa, BMO, $H^{1}$, and Calderón-Zygmund operators for non doubling measures, Math. Ann. 319 (2001), 89-149. MR 1812821 (2002c:42029)

[27] X. Tolsa, Littlewood-Paley theory and the T(1) theorem with non-doubling measures, Adv. in Math. 164 (2001), 57-116. MR.1870513 (2003e:42029)

[28] X. Tolsa, The space $H^{1}$ for nondoubling measures in terms of a grand maximal operator, Trans. Amer. Math. Soc. 355 (2003), 315-348. MR.1928090 (2003e:42030)

[29] X. Tolsa, A proof of the weak $(1,1)$ inequality for singular integrals with non doubling measures based on a Calderón-Zygmund decomposition, Publ. Mat. 45 (2001), 163-174. MR.1829582 (2002d:42019)

[30] H. Triebel, Interpolation Theory, Function Spaces, Differential Operators, the second edition, Johann Ambrosius Barth Verlag, Herdelberg, 1995. MR1328645 (96f:46001)

[31] H. Triebel, Theory of Function Spaces, Birkhäuser Verlag, Basel, 1983. MR0781540 (86j:46026)

[32] H. Triebel, Theory of function spaces, II, Birkhäuser Verlag, Basel, 1992. MR1163193 (93f:46029)

[33] K. Yosida, Functional Analysis, Springer-Verlag, Berlin, 1980. MR0617913 (82i:46002)

Department of Mathematics, Zhongshan University, Guangzhou 510275, People's RePUBLIC OF CHINA

E-mail address: stsdd@zsu.edu.cn

Department of Mathematics, Auburn University, Alabama 36849-5310

E-mail address: hanyong@mail.auburn.edu

School of Mathematical Sciences, Beijing Normal University, Beijing 100875, PeoPLE'S REPUBLiC OF CHINA

E-mail address: dcyang@bnu.edu.cn 